\title{
Open-Loop Stability and Closed-Loop Gust Alleviation on Flexible Aircraft Including Wake Modeling
}

\author{
Joseba Murua, Rafael Palacios, J. Michael R. Graham ${ }^{\ddagger}$ \\ Department of Aeronautics, Imperial College, London, SW7 2AZ, United Kingdom
}

\begin{abstract}
This paper numerically investigates the dynamics of a flexible, lightweight, unmanned aircraft, evaluating its stability boundaries and focusing on the response of the aircraft under atmospheric disturbances. This is achieved by integrating a time-domain 3-D unsteady vortex-lattice aerodynamics method with a geometrically-exact composite beam model encompassing elastic and rigid-body degrees of freedom. The resulting framework is a medium-fidelity tool for the analysis of vehicles that exhibit substantial couplings between their aeroelastic and flight dynamics responses. In its general nonlinear form, the unified model captures the instantaneous shape of the lifting surfaces and the free wake, including large geometrically-nonlinear displacements, in-plane motions, and aerodynamic interference effects. The linearization of the equations leads to a monolithic state-space assembly, ideally suited for stability analysis and control synthesis. The numerical studies illustrate these capabilities, designing linear PID controllers in order to alleviate gust-induced loads and trajectory deviations.
\end{abstract}

\section{Nomenclature}

Symbols

A aerodynamic influence coefficient matrix

AoA angle of attack

$C \quad$ wake-convection matrix

$C^{B a}$ coordinate transformation matrix, from $a$ to $B$

$Q \quad$ global column vector of generalized forces

$\vec{R} \quad$ local position vector along the beam reference line

$t \quad$ physical time

$T \quad$ thrust per propeller

$\vec{v} \quad$ inertial translational velocity of the body-fixed frame, $a$

$\boldsymbol{w} \quad$ column vector of non-circulatory velocities at all collocation points

$\boldsymbol{u}$ input vector

$\boldsymbol{x} \quad$ state vector

$\boldsymbol{y} \quad$ output vector

Greek

$\Gamma \quad$ circulation strength of a vortex ring

$\delta \quad$ control-surface deflection

$\zeta \quad$ coordinates of the aerodynamic lattice

$\eta \quad$ vector of displacements and rotations at all finite element nodes

$\Theta \quad$ Euler angles

${ }^{*}$ Graduate student, AIAA Student Member.

${ }^{\dagger}$ Lecturer, AIAA Member (Contact author. e-mail: rpalacio@imperial.ac.uk).

¥Professor. 


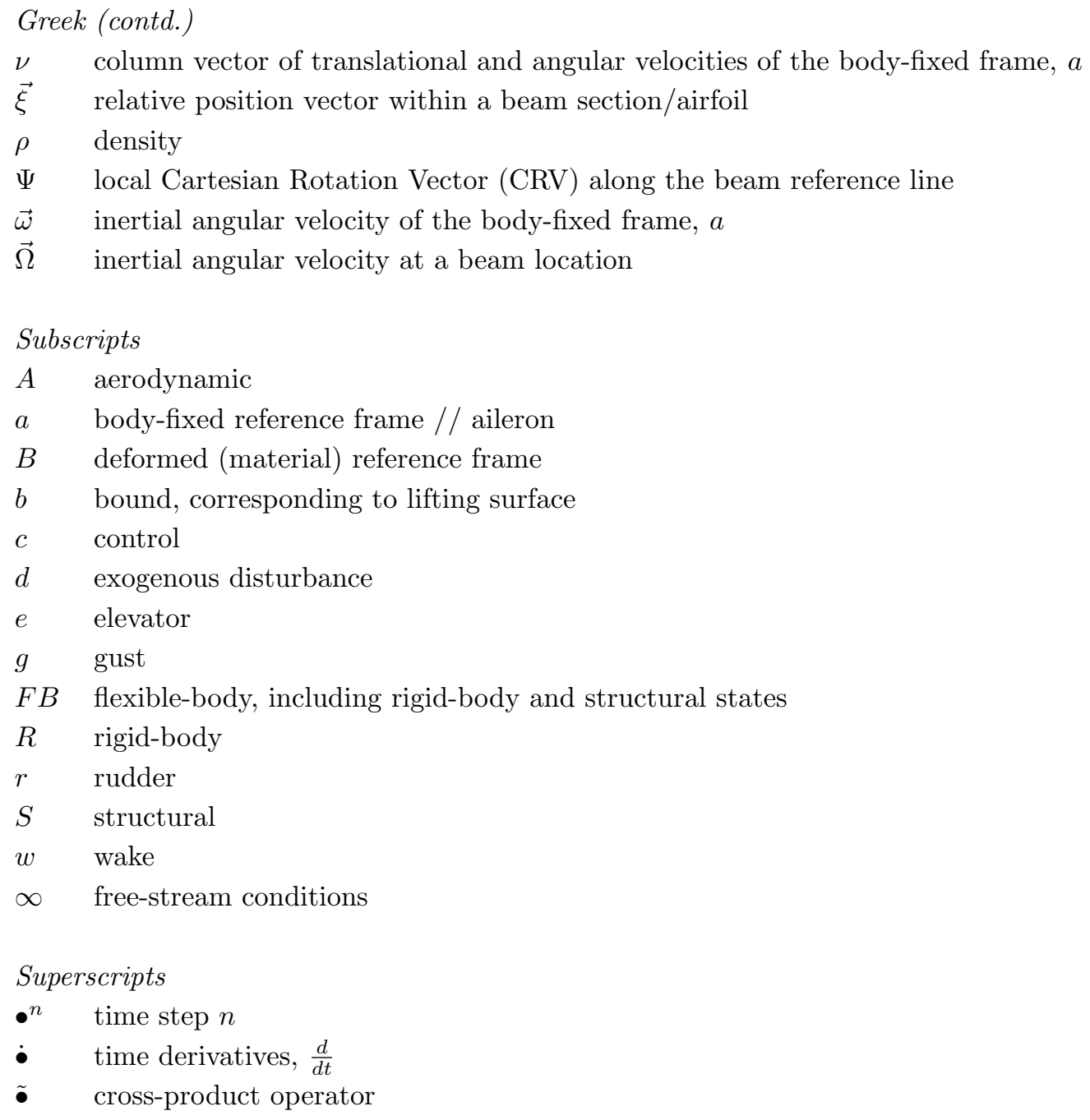

\section{Introduction}

Several prototypes are being developed for solar-powered High-Altitude Long-Endurance (HALE) unmanned flight. For these concepts to become commercially feasible, however, a better understanding of the multidisciplinary interactions between aeroelastic and flight dynamics responses is necessary. New analysis tools that incorporate all relevant physics are required, preferably at low computational cost for early concept assessment and design of appropriate controllers. In particular, the resilience of these flexible Unmanned Aerial Vehicles (UAVs) to atmospheric disturbances has proved to be a crucial factor for their practical operation, ${ }^{1}$ and they still face a major structural integrity risk in adverse weather conditions.

Encounters with gusts and atmospheric turbulence constitute some of the most critical dynamic-load conditions aircraft can experience, ${ }^{2-5}$ and both the sizing of the structure and the design of Flight Control Systems (FCS) are intimately linked to the response under these load cases. For the certification of large commercial airplanes the airworthiness authorities require clearance of continuous turbulence and tuned discrete-gusts, ${ }^{6,7}$ which affect fatigue life of structural members and the maximum stresses they are subject to. The disturbances are evaluated through idealizations, namely a frequency-domain spectral approximation for continuous random turbulence and "1-cos" pulses for discrete gusts. Methods of different fidelity might be employed to represent the aircraft dynamics, but they customarily include gust-penetration effects and model the tail plane. In both continuous and discrete analysis, vertical and lateral disturbances are considered for the relevant mass configurations, flight levels, and Mach numbers. The gust impact on maneuvers might also be accounted for. In all cases, the load alleviation due to the rigid-body motions of the vehicle needs to be considered. ${ }^{8}$

Continuous turbulence is generally analyzed in the frequency domain, assuming isotropy and a statistical 
Gaussian probability distribution, where the Power Spectral Density (PSD) is represented by Dryden ${ }^{9}$ or von Kármán ${ }^{10,11}$ spectra. The PSD and root-mean-square values of the response are then obtained through the relevant aircraft-dynamics model, which, as based in the frequency domain, needs to be linear. Alternatively, rational approximations to the longitudinal and transverse spectra are also possible, which allow time histories of the turbulence to be obtained, ${ }^{12}$ and thus evaluation of the response in the time domain including nonlinearities, such as those of FCS or due to large deformations.

Discrete-gust response is studied in the time domain, and models based on strip theory, for instance, allow nonlinearities to be incorporated. ${ }^{13}$ In contrast, the most common approach in industry is based on the Doublet-Lattice Method (DLM) ${ }^{14}$ which provides the aerodynamic influence coefficients as a function of the reduced frequency, and is therefore linear. In this case, a state-space time-domain representation is obtained via a rational fraction approximation. ${ }^{15-17}$ Further nonlinearities arise if the flight conditions are in the transonic regime, and in this case one would need to resort to CFD analysis. ${ }^{18}$ Regardless of the model used, discrete-gust analysis requires a tuning process in which the critical gust length of the "1-cos" pulse is determined for each set of flight conditions on a set of "Interesting Quantities" (e.g., root bending moment, shear force at engine mounts, etc.).

Large and flexible vehicles are especially vulnerable to atmospheric disturbances, and the response of non-conventional aircraft concepts has been recently addressed by different authors, including flying-wing configurations, ${ }^{13,19,20}$ blended-wing-body airliners, ${ }^{21}$ and flexible HALE platforms. ${ }^{22}$ In order to study Very Flexible Aircraft (VFA) and their behavior under gusts and turbulence, models of different fidelity have been considered. Solution of the Navier-Stokes equations for three-dimensional, unsteady flow-fields with large motions of the solid boundaries is still very demanding, and these field methods have therefore found very limited applications so far in aircraft aeroelasticity and flight dynamics with large wing deformations. ${ }^{23-25}$ As a result, lower-fidelity tools have been overwhelmingly favored over the last years, ${ }^{26-31}$ and these works have allowed the critical influence of the large wing deformations on the aircraft dynamics to be identified.

Motivated by the characteristics slenderness of the airframe structures, the most conventional approach has been to use geometrically-nonlinear beam models for the structural dynamics coupled with 2-D strip theory for the aerodynamics. Nevertheless, previous contributions by the authors showed that these formulations might present some shortcomings likely to play a relevant role in the behavior of the vehicle. On the one hand, the traditional corrections on the lift curve to account for wing-tip aerodynamic effects are only valid in small ranges of reduced frequencies. ${ }^{32}$ On the other, wake-tail interference effects might affect the open-loop and stability characteristics of these platforms. ${ }^{33-35}$

HALE aircraft are large vehicles flying at low speeds that are likely to satisfy potential-flow assumptions, hence rendering panel methods excellent candidates for the description of the aerodynamics: without incurring excessive computational costs, they can incorporate 3-D effects, interference and wake modeling. As a drawback, panel methods are not appropriate when the wing enters stalled conditions, to predict drag, or at very low Reynolds number. Neither are they adequate at very high altitudes, potentially within the range of operations of HALE UAVs, due to dominance of rarefied flow effects. Note however that the critical load conditions will occur during climb and descent operations in the lower atmosphere, which is the scope of aeroelastic analysis.

Among unsteady panel methods, the Doublet-Lattice Method is hindered by the fact that its standard form is limited to out-of-plane small-amplitude motions. In turn, the Unsteady Vortex-Lattice Method (UVLM) can provide a powerful alternative in many scenarios, since the general formulation caters for geometrically-nonlinear deflections of the lifting surfaces by imposing the impermeability boundary-condition on the deformed shape, and includes a representation of a free wake. The linearized version of the equations leads to a convenient and efficient state-space form, which could substitute the DLM in virtually any aeroelastic analysis offering the same degree of fidelity. It can be further employed in situations where more complex motions need modeling, including in-plane motions, non-planar wakes, and/or small oscillations around large static deformations.

As a result, the UVLM will be used in this work to address the medium-fidelity modeling and simulation of low-speed flexible UAVs. The aerodynamic model (Section II) is coupled with a Geometrically-Exact Composite Beam description (Section III) for a fully-coupled description of the flexible-aircraft dynamics (Section IV). For the numerical results (Section V) a representative HALE configuration is defined, and the impact of flexibility on its stability characteristics is illustrated first. Then the paper focuses on the openand closed-loop response of the aircraft under atmospheric disturbances, implementing proportional-integralderivative (PID) feedback control in the linear model for both load alleviation and trajectory regulation. 


\section{Unsteady Vortex-Lattice Method}

The basics of the Unsteady Vortex-Lattice algorithm are described in excellent detail by Katz and Plotkin. ${ }^{36}$ Here, the general nonlinear governing equations are presented in discrete-time state-space fashion, which is found to be more suitable for multidisciplinary integration and aircraft dynamics modeling. The nonlinearities arise due to the enforcement of the boundary conditions on the deformed geometry and the free-wake model. As main focus of the results of this paper, the linearized form of the state-space equations is also subsequently derived by performing small perturbation expansions and assuming a frozen aerodynamic geometry, i.e., flow tangency is enforced on the reference configuration and the wake convects with the free-stream velocity. This formulation follows upon the concepts outlined by Hall. ${ }^{37}$
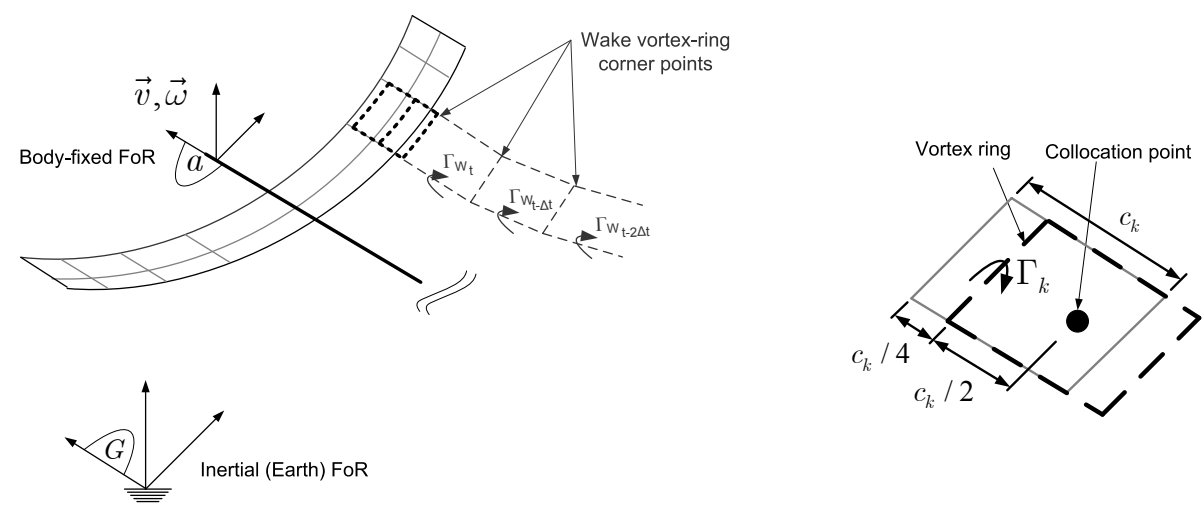

Figure 1. Unsteady aerodynamics model: lifting-surface and wake discretization using vortex-ring elements.

\section{A. General nonlinear formulation}

In the UVLM, rectilinear vortex-ring elementary solutions are distributed over the mean surface, ignoring thickness. The leading segment of the vortex ring lies on the panel's quarter-chord line, and a collocation point is placed at the three-quarter chord line, which falls at the center of the vortex ring (see Figure 1). As the surface moves along its flight path, a force-free wake is obtained as part of the solution procedure, also represented by vortex rings. By imposing the non-penetration boundary condition at a number of collocation points, a system of algebraic equations is obtained. Thus, at discrete time step $n+1$, the vorticity distribution of the bound vortex elements is determined by

$$
A_{c b} \boldsymbol{\Gamma}_{b}^{n+1}+A_{c w} \boldsymbol{\Gamma}_{w}^{n+1}+\boldsymbol{w}^{n+1}=0,
$$

where $\boldsymbol{\Gamma}_{b}$ and $\boldsymbol{\Gamma}_{w}$ are the column vectors with the circulation strengths in the bound and wake vortex-rings, respectively; $A_{c b}=A_{c b}\left(\boldsymbol{\zeta}_{b}^{n+\epsilon}\right)$ and $A_{c w}=A_{c w}\left(\boldsymbol{\zeta}_{b}^{n+\epsilon}, \boldsymbol{\zeta}_{w}^{n+\epsilon}\right)$ are the wing-wing and wing-wake aerodynamic influence coefficient matrices, computed at the collocation points through the Biot-Savart law, and $\boldsymbol{\zeta}_{b}$ and $\boldsymbol{\zeta}_{w}$ are the column vectors with the bound and wake grid coordinates. $\boldsymbol{w}$ in Eq. (1) is the column vector of normal components of all velocities except those induced by bound and wake vorticity, which may encompass deployment of control surfaces, gust induced velocities, wing deformations and rigid-body motions, and will be denoted here as "non-circulatory velocity". The time at which the different variables are evaluated within the current time step is determined by $t^{n+\epsilon}=t^{n}+\epsilon \Delta t$, with $0 \leq \epsilon \leq 1$, and depends on the integration scheme.

At each time step, as the circulation of the wing changes, a new row of vortex rings will be shed into the wake from the trailing edge of each lifting surface. In addition to this, the existing wake may be displaced following the local flow velocity (the free-wake model). This is written as

$$
\boldsymbol{\zeta}_{w}^{n+1}=C_{\zeta b} \boldsymbol{\zeta}_{b}^{n+1}+C_{\zeta w} \boldsymbol{\zeta}_{w}^{n}+\int_{t^{n}}^{t^{n+1}} \boldsymbol{V}(t) \mathrm{d} t
$$

where $C_{\zeta b}$ and $C_{\zeta w}$ are very sparse constant convection matrices that update the position of the prescribed wake: $C_{\zeta b}$ closes the newly shed wake panel with the trailing edge of the lifting surface, satisfying the KuttaJoukowski condition, while $C_{\zeta w}$ preserves the wake of the previous time step unchanged. The column vector 
$\boldsymbol{V}$ in Eq. (2) includes the local induced flow velocities at the grid points of the wake mesh. If a prescribed wake were to be considered, the integral term would be dropped, but for a fully force-free wake it is necessary to retain it and time-integration is required to determine its location. Conventionally, this is done using an explicit one-step Euler method, but in order to improve the accuracy and/or stability of the wake roll-up other higher-order schemes have been also proposed in the literature, such as a two-step Euler ${ }^{38}$ and the fourth-order Adams-Bashforth-Moulton. ${ }^{39}$

In turn, the propagation equation for the wake circulation can be written in discrete time as

$$
\boldsymbol{\Gamma}_{w}^{n+1}=C_{\Gamma b} \boldsymbol{\Gamma}_{b}^{n}+C_{\Gamma w} \boldsymbol{\Gamma}_{w}^{n}
$$

where $C_{\Gamma b}$ and $C_{\Gamma w}$ map the circulation of the previous time step to the current one, and they are very sparse constant matrices which account for Kelvin's circulation theorem (that enforces the condition for wake shedding at the trailing edge) and Helmholtz's vortex theorem (in the convection of the wake). As the influence of the wake decreases very rapidly as it is convected away from the lifting surface (due to the Biot-Savart law), the computational burden can be significantly alleviated by neglecting the influence of remote panels (wake truncation). Implementing dissipation models has also been tried ${ }^{40,41}$ but in these cases consideration must be given to conservation of circulation issues.

Finally, once the distribution of vorticity has been obtained at each time step, the inviscid aerodynamic loads can be computed. The pressure differential, obtained from the unsteady Bernoulli equation, acts along the normal vector of each panel. However, as the UVLM is based on thin-wing theory, it does not account for the leading-edge suction, ${ }^{42}$ and only the contribution to the local lift is kept, i.e., the force normal to the local velocity. The contribution of the panel to the induced drag can be computed through the approximation proposed by Katz and Plotkin. ${ }^{36}$ As a result, these inviscid loads can be written as

$$
\begin{aligned}
\boldsymbol{L}^{n} & =\rho_{\infty} G_{c}\left[\left(U_{c} \Lambda_{c}+U_{s} \Lambda_{s}\right) \boldsymbol{\Gamma}_{b}^{n}+\dot{\boldsymbol{\Gamma}}_{b}^{n}\right], \\
\boldsymbol{D}^{n} & =\rho_{\infty}\left[-\hat{U} \Lambda_{c} \boldsymbol{\Gamma}_{b}^{n}+G_{s} \dot{\boldsymbol{\Gamma}}_{b}^{n}\right],
\end{aligned}
$$

where $\Lambda_{c(s)}$ are matrices filled with 1 and -1 in the correct positions in order to account for adjacent panels; matrices $G_{c(s)}=G_{c(s)}\left(\boldsymbol{\zeta}_{b}^{n}, \dot{\boldsymbol{\zeta}}_{b}^{n}\right)$ are diagonal matrices dependent on the panel geometry and local angle of incidence; $U_{c(s)}=U_{c(s)}\left(\boldsymbol{\Gamma}_{w}^{n}, \boldsymbol{\zeta}_{b}^{n}, \boldsymbol{\zeta}_{w}^{n}, \dot{\boldsymbol{\zeta}}_{b}^{n}\right)$ and $\hat{U}=\hat{U}\left(\boldsymbol{\Gamma}_{b}^{n}, \boldsymbol{\Gamma}_{w}^{n}, \boldsymbol{\zeta}_{b}^{n}, \boldsymbol{\zeta}_{w}^{n}\right)$ are diagonal matrices that store weighted velocities. ${ }^{43}$

\section{B. Linearization assuming a frozen geometry}

The above equations correspond to the general formulation of the UVLM, which can account for large deflections of the lifting surfaces in unsteady flow. In many cases, however, it will be useful to look at the linearized form of these equations, when relative deformations are small around an equilibrium condition, for problems such as stability analysis and design of linear control laws. In this context, the equilibrium condition will generally correspond to a trimmed aircraft configuration, which can exhibit large static deformations. This reference configuration represents the initial conditions for impulsive motions, and the mean conditions for periodic oscillations. The linearization of the unsteady aerodynamics, Eqs. (1-4), is carried out in bodyfixed axes (as opposed to the usual stability axes in flight dynamics ${ }^{44}$ ), assuming small dynamic excursions around the statically deformed aircraft and therefore a frozen aerodynamic geometry. The latter has two main implications:

- For the aerodynamic grid, $\boldsymbol{\zeta}_{b}$, the dependencies on position are neglected. However, orientation changes as lifting surfaces deform are actually included, since these are necessary to account for variations in local angle of incidence and static loading, and rotation of control surfaces. This dependency on orientation will be introduced via the derivatives of the follower forces in the structural model presented in Section III. Note also that the dependency on velocities, $\dot{\boldsymbol{\zeta}}_{b}$, must be catered for.

- Free-wake effects around the reference condition are neglected. This assumption reduces the UVLM to a prescribed-wake method, and under this approximation it is not necessary to keep track of the wake shape after trim as it conforms to free-stream convection alone. Note that the wake is prescribed in this case, but it does not need to be flat, and it will be shed from the deformed lifting surface. Besides, a rolled-up wake can be considered in order to determine equilibrium more accurately (in a steady sense). As the wake is 
frozen, there is no need to retain Eq. (2). Besides, $\boldsymbol{\zeta}_{w}$ will remain constant at the reference configuration and is no longer a state of the system.

Performing a small perturbation Taylor expansions on the general UVLM equations, the linearized (incremental) propagation equations are obtained. These define the descriptor state-space form of the linear UVLM as ${ }^{45}$

$$
\begin{aligned}
E_{A} \Delta \boldsymbol{x}_{A}^{n+1}+F_{A} \Delta \boldsymbol{u}_{A}^{n+1} & =A_{A} \Delta \boldsymbol{x}_{A}^{n}+B_{A} \Delta \boldsymbol{u}_{A}^{n}, \\
\Delta \boldsymbol{y}_{A}^{n} & =C_{A} \Delta \boldsymbol{x}_{A}^{n}+D_{A} \Delta \boldsymbol{u}_{A}^{n},
\end{aligned}
$$

where the outputs $\boldsymbol{y}_{A}$ are the aerodynamic loads, Eq. (4), and the states and inputs that fully define the linearized aerodynamic system are

$$
\boldsymbol{x}_{A}=\left\{\begin{array}{c}
\boldsymbol{\Gamma}_{b} \\
\boldsymbol{\Gamma}_{w} \\
\dot{\boldsymbol{\Gamma}}_{b}
\end{array}\right\}, \quad \text { and } \quad \boldsymbol{u}_{A}=\left\{\begin{array}{c}
\boldsymbol{\zeta}_{b} \\
\dot{\boldsymbol{\zeta}}_{b}
\end{array}\right\}
$$

This descriptor form of the equations, with matrix $E_{A}$ pre-multiplying the updated value on the state variable is the natural expression obtained from the UVLM. The linearized state-space UVLM provides a compact and efficient tool, of comparable fidelity to the DLM, but without some of its restrictions: the wake can be nonplanar, flow tangency is imposed on the statically deformed geometry, and in-plane deformations are captured. Besides, it will be shown in Section IV.C that it enables the incorporation of rigid-body motions and elastic deformations in a unified monolithic framework.

\section{Geometrically-Exact Composite Beam Model}

The primary aircraft structures (wing, fuselage, empennage) are modeled as composite curvilinear beams capable of large deflections and global rotations, but under the assumption of small local strains. ${ }^{46,47}$ They undergo three-dimensional displacements and rotations, with cross-sectional properties calculated along the span. Typically those displacements and rotations are the primary variables in the numerical solution of the structural problem, ${ }^{48,49}$ but alternative solutions exists using the strain of the beam elements as primary variables, ${ }^{30,50,51}$ or taking both the local velocities and strains (the intrinsic description) as independent degrees of freedom. ${ }^{52-54}$ A comparative study on these different structural models for flexible-aircraft dynamics $^{32}$ showed that even though the intrinsic model is in general numerically more efficient, some of the advantages disappear when run in combination with an aerodynamic model. In those situations, the displacement-based approach might be preferable, since the description is more intuitive, easily linearizable, and naturally incorporates displacement constraints in closed kinematic chains.
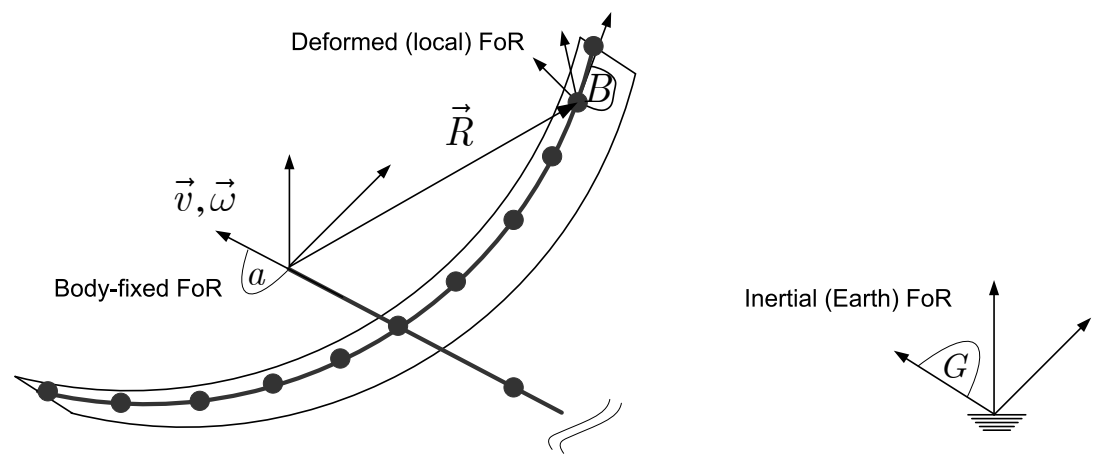

Figure 2. Flexible-body model: geometrically-exact beam elements.

As a consequence, nodal displacements, $\vec{R}$, and the Cartesian Rotation Vector (CRV), $\Psi$, are taken as primary degrees of freedom in this work. There are no constraints on the undeformed configuration allowing the beam to be initially curved and twisted. As shown in Figure 2, the vehicle dynamics are described by 
a body-fixed frame of reference, $a$, which moves with respect to an inertial frame, $G$, by the translational, $v_{a}(t)$, and angular, $\omega_{a}(t)$, velocities of its origin - subscripts are used to indicate the coordinate system in which each vector magnitude is projected. The orientation of the body-fixed frame with respect to the inertial one is given by the coordinate transformation matrix $C^{G a}(t)$, determined via Euler angles, $\Theta$, and a flat Earth is assumed. The local orientation of the beam cross sections (airfoils) is defined by their local coordinate systems, $B$, in the deformed (or current) configuration.

The equations of motion are obtained from Hamilton's principle, ${ }^{32}$ for which the potential, $\mathcal{U}$, and kinetic, $\mathcal{T}$, energy densities per unit length are first computed as

$$
\mathcal{U}=\frac{1}{2}\left\{\begin{array}{ll}
\gamma^{T} & \kappa^{T}
\end{array}\right\} \mathcal{S}\left\{\begin{array}{l}
\gamma \\
\kappa
\end{array}\right\}, \text { and } \mathcal{T}=\frac{1}{2}\left\{\begin{array}{ll}
V_{B}^{T} & \Omega_{B}^{T}
\end{array}\right\} \mathcal{M}\left\{\begin{array}{c}
V_{B} \\
\Omega_{B}
\end{array}\right\} .
$$

Here, $V_{B}$ and $\Omega_{B}$ are the inertial velocities of the local deformed frame, $\gamma$ and $\kappa$ are the beam strains, and $\mathcal{M}$ and $\mathcal{S}$ are the mass and stiffness matrices, respectively, which are obtained through an appropriate cross-sectional analysis methodology. ${ }^{55,56}$ The orientation of cross-sections at each point in the current configuration is described in terms of finite rotations from the body-fixed reference frame, $a$, to the local deformed frame, $B$, using the CRV, $\Psi(s, t)$. The corresponding coordinate transformation matrix will be $C^{B a}(\Psi)$. Strains and velocities can then be expressed in terms of the independent set of variables, $R_{a}(s, t)$ and $\Psi(s, t) .{ }^{46,49}$ This general description of the beam dynamics is independent of any discretization used. Here, the position and rotation vectors are approximated using finite elements with linear interpolation. If $\boldsymbol{\eta}$

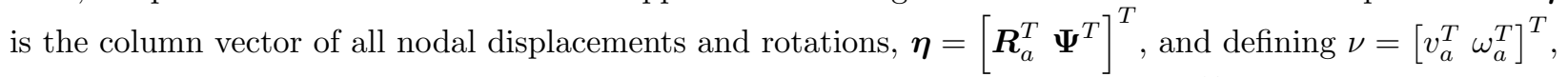
the equations of motion of the aircraft can be cast into the following discrete form ${ }^{32}$

$$
M(\boldsymbol{\eta})\left\{\begin{array}{l}
\ddot{\boldsymbol{\eta}} \\
\dot{\nu}
\end{array}\right\}+Q_{g y r}(\boldsymbol{\eta}, \dot{\boldsymbol{\eta}}, \nu)+Q_{s t i f}(\boldsymbol{\eta})=Q_{e x t}\left(\boldsymbol{\eta}, \dot{\boldsymbol{\eta}}, \nu, \Theta, \boldsymbol{u}_{F B}\right),
$$

where matrix $M$ is the tangent mass matrix and $Q_{g y r}, Q_{\text {stiff }}$ and $Q_{e x t}$ are the discrete gyroscopic, stiffness, and external generalized forces, respectively. The input vector $\boldsymbol{u}_{F B}=\left[\boldsymbol{u}_{S}^{T} \mid \boldsymbol{u}_{R}^{T}\right]^{T}$ includes the dependency of the external loads in the most general form. In particular, for the aeroelastic and flight dynamics analysis, $\boldsymbol{u}_{F B}$ will include control-surface inputs, gusts and aerodynamic states.

The linearized (incremental) form of Eq. (8) around an equilibrium point is given by

$$
M\left(\boldsymbol{\eta}_{o}\right)\left\{\begin{array}{c}
\Delta \ddot{\boldsymbol{\eta}} \\
\Delta \dot{\nu}
\end{array}\right\}+C\left\{\begin{array}{c}
\Delta \dot{\boldsymbol{\eta}} \\
\Delta \nu
\end{array}\right\}+K\left\{\begin{array}{c}
\Delta \boldsymbol{\eta} \\
0
\end{array}\right\}=\Delta Q_{\text {ext }},
$$

where $C$ and $K$ are the tangent damping and stiffness matrices, evaluated at the equilibrium point.

The $2^{\text {nd }}$ order Ordinary Differential Equations that describe the flexible-body dynamics, Eq. (8) for the nonlinear problem and Eq. (9) for the linear one, will be discretized in time using the standard Newmark- $\beta$ $\operatorname{method}^{57}$ - other temporal discretizations of the equations of motion have also been suggested, such as the Generalized- $\alpha .^{30,58}$ In particular, the linearized equations, Eq. (9), can be wrapped up in implicit state-space form after temporal discretization as ${ }^{45}$

$$
\begin{gathered}
E_{F B}\left\{\begin{array}{c}
\Delta \boldsymbol{x}_{S} \\
\Delta \boldsymbol{x}_{R}
\end{array}\right\}^{n+1}+F_{F B}\left\{\begin{array}{c}
\Delta \boldsymbol{u}_{S} \\
\Delta \boldsymbol{u}_{R}
\end{array}\right\}^{n+1}=A_{F B}\left\{\begin{array}{l}
\Delta \boldsymbol{x}_{S} \\
\Delta \boldsymbol{x}_{R}
\end{array}\right\}^{n}+B_{F B}\left\{\begin{array}{l}
\Delta \boldsymbol{u}_{S} \\
\Delta \boldsymbol{u}_{R}
\end{array}\right\}^{n}, \\
\left\{\begin{array}{l}
\Delta \boldsymbol{y}_{S} \\
\Delta \boldsymbol{y}_{R}
\end{array}\right\}^{n}=\left\{\begin{array}{l}
\Delta \boldsymbol{x}_{S} \\
\Delta \boldsymbol{x}_{R}
\end{array}\right\}^{n},
\end{gathered}
$$

where $\boldsymbol{y}_{S}$ and $\boldsymbol{y}_{R}$ are the output states of the structural and rigid-body equations, respectively, and the states that fully determine the flexible-body dynamics are

$$
\boldsymbol{x}_{F B}=\left[\boldsymbol{x}_{S}^{T} \mid \boldsymbol{x}_{R}^{T}\right]^{T}=\left[\boldsymbol{\eta}^{T} \dot{\boldsymbol{\eta}}^{T} \mid \nu^{T} \Theta^{T}\right]^{T} .
$$

It has been assumed that the output is the full deformed state. This will lead to a linear state-space monolithic description of the aeroelastic and flight dynamics coupled problem, as elucidated in Section IV.C. 


\section{Flexible-Aircraft Dynamics: Integration of Aeroelasticity and Flight Dynamics}

In traditional aircraft dynamics analyses aeroelasticity and flight mechanics are studied as independent blocks stemming from the significant difference in the times scales of elastic and rigid-body modes. However, this procedure is not suitable for moderately-to-highly flexible vehicles, since structural frequencies might overlap with those of flight dynamics. A substantial effort has been recently carried out in order to build unified frameworks that incorporate flexible-multibody dynamics and potential-flow aerodynamics, ${ }^{28-30,59,60}$ but these have been usually restricted to two-dimensional aerodynamics or linearized boundary conditions.

Coupling the Unsteady Vortex-Lattice Method (Section II) with a geometrically-nonlinear beam (Section III) overcomes some of the limitations of the above models, and allows fully nonlinear time-marching simulations and linearized monolithic state-space solutions. The mapping between discretizations and different types of integration are presented next.

\section{A. Mapping}

As the flexible-body model is based on beams (curves in space) and the aerodynamic lattice is distributed over a lifting surface, a mapping procedure is required between both meshes. This is accomplished here by assuming that the lifting-surface cross-sections remain undeformed, that the finite-element discretization of the beam coincides with the spanwise aerodynamic grid, and that the aerodynamic loads can be approximated as isolated forces acting on the center of the leading segment of each vortex-ring. ${ }^{35}$

Firstly, displacements and rotations of the beam nodes, $\boldsymbol{R}_{a}$ and $\boldsymbol{\Psi}$, and the corresponding rates, $\dot{\boldsymbol{R}}_{a}$ and $\dot{\boldsymbol{\Psi}}$, have to be transformed to deformations and velocities of the grid points of the aerodynamic lattice, $\boldsymbol{\zeta}_{b}$ and $\dot{\boldsymbol{\zeta}}_{b}$. For the vortex-ring vertices, the transformations of the relative position with respect to the body-fixed frame, $\left(\zeta_{b}\right)_{a}$, and inertial velocity expressed in the $a$ frame, $\left(\dot{\zeta}_{b}\right)_{a}$, are given by

$$
\begin{aligned}
\left(\zeta_{b}\right)_{a} & =R_{a}+C^{a B} \xi_{B}, \\
\left(\dot{\zeta}_{b}\right)_{a} & =v_{a}+\widetilde{\omega}_{a} R_{a}+\dot{R}_{a}+C^{a B} \widetilde{\Omega}_{B} \xi_{B},
\end{aligned}
$$

where $\xi_{B}$ is the (constant) distance between a vortex-ring corner point and the relevant node, and $\Omega_{B}=$ $T(\Psi) \dot{\Psi}+C^{B a}(\Psi) \omega_{a}$ is the local inertial angular velocity, with $T(\Psi)$ the tangential operator. ${ }^{52}$ Positions and velocities of the collocation points are obtained through linear interpolation.

Secondly, the inviscid aerodynamic forces computed in Eq. (4) are converted into forces and moments acting upon the beam nodes. They are expressed in the local aerodynamic frame, $A$, defined by the instantaneous non-circulatory velocity, and thus need to be transformed to the body-fixed $a$ frame, in order to be consistent with the flexible-beam equations, Eq. (8). Finally, they are lumped into the nodes of the deformed beam, splitting them between adjacent ones. Integration of these nodal values yields the resultant forces and moments on the body-fixed frame. These operations can be summarized as

$$
\boldsymbol{Q}_{a}^{S}=\left\{\begin{array}{c}
\hat{\boldsymbol{F}}_{a} \\
\hat{\boldsymbol{M}}_{a}
\end{array}\right\}=\chi_{v r \rightarrow n o} \bar{C}^{a A} \boldsymbol{F}_{A}, \text { and } Q_{a}^{R}=\chi_{n o \rightarrow b f} \boldsymbol{Q}_{a}^{S},
$$

where $\bar{C}^{a A}\left(\boldsymbol{\zeta}_{b}, \dot{\zeta}_{b}\right)$ is a block diagonal matrix, being each block given by the corresponding coordinate transformation matrix from the local aerodynamic to the body-fixed frame, $C^{a A}$. In turn, $\chi_{v r \rightarrow n o}=$ $\chi_{v r \rightarrow n o}\left(\boldsymbol{R}_{a}, \Psi, \boldsymbol{\zeta}_{b}\right)$ is a very sparse matrix that lumps the forces acting on the aerodynamic lattice, expressed in $a$, into forces and moments applied on the beam nodes, and $\chi_{n o \rightarrow b f}=\chi_{n o \rightarrow b f}\left(\boldsymbol{R}_{a}, \boldsymbol{\Psi}\right)$ integrates the nodal forces and moments to give the resultant loads at the body-fixed frame.

The linearized form of these fluid/structure mappings for grid and loads is again obtained through small perturbation analysis, under the assumptions outlined in Section II.B. They can be written as ${ }^{45}$

$$
\begin{aligned}
& \Delta \boldsymbol{u}_{A S}^{n}=p_{A S}^{n} P_{A S} \Delta \boldsymbol{y}_{S}^{n}, \quad \text { and } \quad \Delta \boldsymbol{u}_{A R}^{n}=p_{A R}^{n} P_{A R} \Delta \boldsymbol{y}_{R}^{n} \text {, } \\
& \Delta \boldsymbol{u}_{S A}^{n}=p_{S A}^{n} P_{S A} \Delta \boldsymbol{y}_{A}^{n}, \quad \text { and } \quad \Delta \boldsymbol{u}_{R A}^{n}=p_{R A}^{n} P_{R A} \Delta \boldsymbol{y}_{A}^{n} \text {, }
\end{aligned}
$$

where capital $P$ matrices represent the actual mapping and lower-case $p^{n}$ scalar values depend on the parameters of the Newmark- $\beta$ and the time step. 


\section{B. Nonlinear time marching}

The nonlinear flexible-aircraft dynamics equations, given by the aerodynamic and the flexible-body ones, Eqs. (1-4) and (8) respectively, are solved using a partitioned time-marching scheme. ${ }^{61}$ Strongly- and weaklycoupled solutions can be implemented, depending on whether a subiteration routine to converge aerodynamics and beam dynamics is included. The strong coupling might offer higher accuracy and better numerical stability properties. However, the weakly-coupled approach is found to work well with the appropriate selection of simulation parameters, and leads to a smaller computational burden.

This fully nonlinear time-marching solution accounts for large geometry changes, both in the structure and in the 3-D aerodynamics, by updating the relevant inertia, gyroscopic and stiffness terms in the structural model, and by enforcing the aerodynamic boundary conditions at the instantaneous deformed shape. In addition, the model includes an inviscid representation of the free aircraft-wake. Ad hoc solutions for dynamic stall have also been suggested. ${ }^{20,62,63}$ This methodology, though necessary for very flexible structures and/or large rigid-body motions, ${ }^{33-35,64}$ is computationally intensive compared to linear potential-flow solvers, and does not lend itself to an accessible formulation for stability analysis or FCS design. To that goal, the linearized version of the coupled equations for closed-loop problems is presented next, which constitutes the main novelty in this paper.

\section{Monolithic integration of linearized equations}

The linearized flexible-aircraft dynamics equations are obtained by assembling together the linearized UVLM, Eq. (5), with the linearized flexible-body equations, Eq. (10), and using the linear interface between both models, Eq. (14). The resulting system can be cast into a monolithic discrete-time state-space formulation, which for the homogeneous problem has the form

$$
E_{\text {sys }} \Delta \boldsymbol{x}^{n+1}=A_{\text {sys }} \Delta \boldsymbol{x}^{n}
$$

where the entries to matrices $E_{\text {sys }}$ and $A_{\text {sys }}$ depend on the equilibrium conditions, and the state vector that completely determines the linear system is

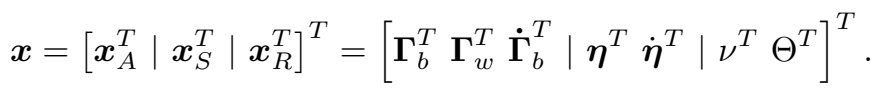

A discrete-time generalized eigenvalue problem can be derived from the homogeneous system equation, Eq. (15). This can be solved directly, without pre-computing aerodynamic forces in the frequency domain or projecting on the structural modes, yielding a very powerful formulation for stability boundary prediction. The coupling is monolithic in this case since the interface between aerodynamics and flexible body-dynamics has been determined analytically. The linearization might take place about a highly deformed configuration, and although the wake is frozen, interference-induced unsteady downwash effects are modeled. Note that a geometrically-nonlinear beam model was used to introduce nonlinear static equilibrium conditions, but the analysis could be also based on the linear normal modes of the deformed structure.

On the other hand, the formulation lends itself to time marching, reduced order modeling, and appending gusts and controls. For instance, in the numerical studies (Section V), feedback PID controllers will be introduced for gust alleviation. In this case the homogeneous problem shown in Eq. (15) is augmented as

$$
\begin{aligned}
E_{\text {sys }} \Delta \boldsymbol{x}^{n+1} & =A_{\text {sys }} \Delta \boldsymbol{x}^{n}+B_{d} \Delta \boldsymbol{u}_{d}^{n}+B_{c} \Delta \boldsymbol{u}_{c}^{n}, \\
\Delta \boldsymbol{y}^{n} & =C \Delta \boldsymbol{x}^{n}, \\
\Delta \boldsymbol{u}_{c}^{n} & =K_{p} \boldsymbol{e}^{n}+K_{i} \Delta t \sum_{k=0}^{n} \boldsymbol{e}^{k}+\frac{K_{d}}{\Delta t}\left(\boldsymbol{e}^{n}-\boldsymbol{e}^{n-1}\right),
\end{aligned}
$$

where $\boldsymbol{u}_{d}$ represents exogenous atmospheric disturbances and $\boldsymbol{u}_{c}$ control inputs ${ }^{\mathrm{a}} . K_{p}, K_{i}$, and $K_{d}$ are, respectively, the matrices with the proportional, integral, and derivative gains of the PID controllers, and $\boldsymbol{e}=\boldsymbol{r}-\Delta \boldsymbol{y}$ is the error column-vector, with $\boldsymbol{r}$ the reference signal. A constant time step $\Delta t$ is assumed. Figure 3 illustrates the assembly of the closed-loop equations

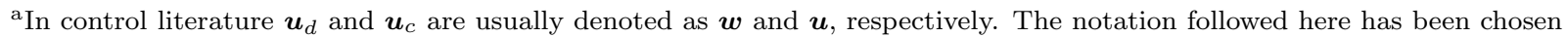
in order to avoid confusion with $\boldsymbol{w}$ in Eq. (1).
} 


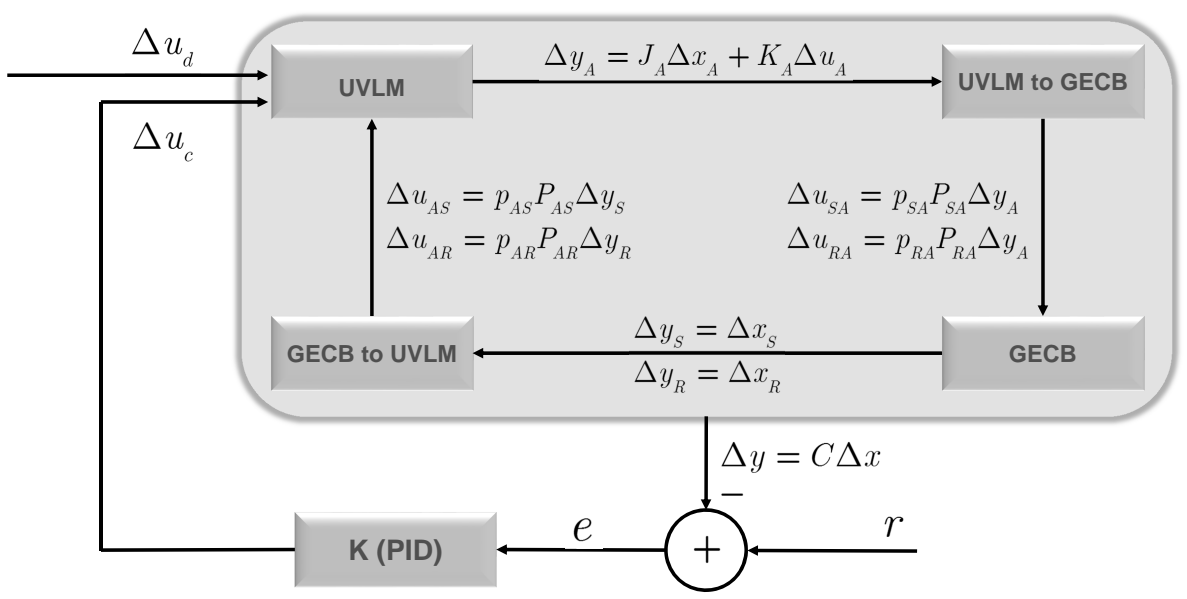

Figure 3. Assembly of linearized flexible-aircraft dynamics equations. Closed-loop using PID control.

\section{Numerical studies}

The methods described above have been implemented in a framework for the Simulation of High-AspectRatio Planes (SHARP). SHARP allows running independently the flexible-body dynamics and aerodynamics solvers, as well as the coupled system. Other non-aerodynamic external forces can be included in the analysis, and specifically gravity is always accounted for. Extensive verification of the tools has been carried out and as these results, among others, have been reported elsewhere, ${ }^{32-35,43,64}$ they will not be repeated here. In turn, this section will illustrate the capabilities of the novel linearized state-space formulation.

For this purpose, a representative flexible UAV has been defined (Section V.A), and its linear stability characteristics are computed first (Section V.B), in order to expose some of its dynamic features and to guarantee that it is open-loop stable. The paper then focuses on open- and closed-loop response of the aircraft when it encounters atmospheric disturbances. Discrete longitudinal gusts (Section V.C) and nonuniform thermal distributions (Section V.D) are studied, and in both cases PID control is implemented to drive the closed-loop behavior.

\section{A. Definition of test case}

The test case used for the numerical results is shown in Figure 4, which has been defined to resemble the latest version of QinetiQ's Zephyr. The vehicle consists of large aspect ratio flexible wings, a rigid fuselage and a rigid empennage. The main wing encompasses rigidly-linked members, with a 10 deg dihedral angle at both ends. The outboard members also include full-span trailing-edge ailerons of a quarter-chord length.

The horizontal stabilizer and the vertical fin are modeled as lifting surfaces, and they also include quarterchord-length control surfaces, elevator and rudder, respectively. The fuselage, in turn, is represented by a non-lifting beam, and it is inclined so that the empennage is raised with respect to the main wing. This feature is important in order to guarantee that the wake shed by the main wing does not impinge on the tail at steady level flight or as the aircraft undergoes small-to-moderate gust-induced motions - the UVLM can provide some insight into wake-tail collisions, ${ }^{35}$ but this is a scenario to be avoided.

All lifting surfaces are uncambered, untwisted, unswept, and untapered. The aircraft carries a payload of $50 \mathrm{~kg}$ at the root of the main wing (the only non-structural mass of the problem, modeled as a point load), and is powered by two massless propellers, which are modeled as point forces rigidly linked to the wing.

The relevant properties of the members are listed in Table 1. The location of elastic axes and centers of gravity has been chosen in order to magnify aeroelastic effects. The mass per unit length of the fuselage is the same as that of the Horizontal Tail Plane (HTP) and Vertical Tail Plane (VTP), and thus the total mass of this aircraft, including payload and structural mass, is $75.4 \mathrm{~kg}$. It is assumed that the aircraft flies at an altitude of $20 \mathrm{~km}$, where the density is $\rho_{\infty}=0.0899 \mathrm{~kg} / \mathrm{m}^{3}$.

Throughout the subsequent numerical studies, a nondimensional time step of $\Delta t^{*}=V_{\infty} \Delta t / c=0.25$ has been used, where $c$ is the characteristic chord length. All lifting surfaces have been discretized using 4 
panels/elements chordwise. 32 spanwise panels/elements are used in the main wing, 8 in the HTP, and 4 in the VTP. The fuselage is represented with 10 elements.

(a)

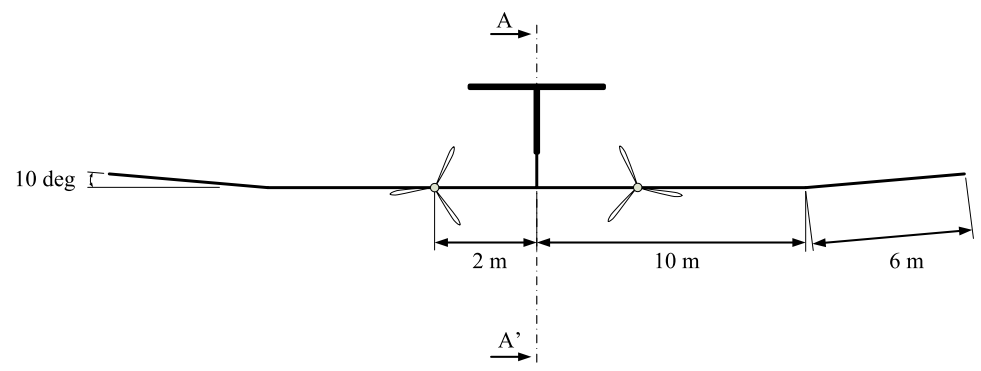

(b)

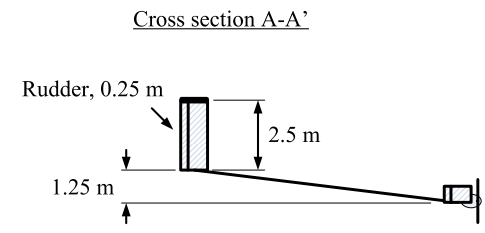

(d)

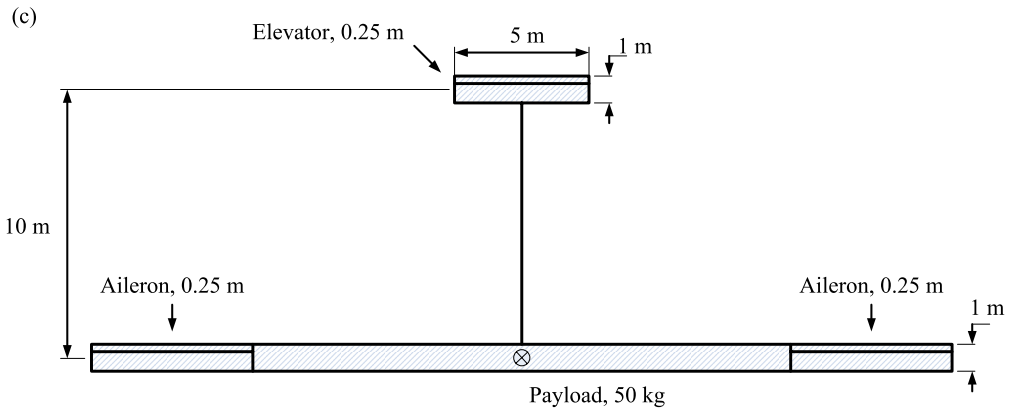

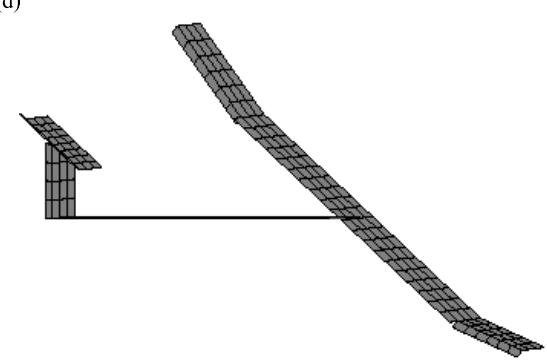

Figure 4. Undeformed test aircraft geometry: (a) front view, (b) cross section, (c) top view, and (d) 3-D view of the aircraft showing deployed control surfaces.

Table 1. Test aircraft properties.

\begin{tabular}{llll}
\hline \hline & Main wing & HTP & VTP \\
\hline Chord & $1 \mathrm{~m}$ & $1 \mathrm{~m}$ & $1 \mathrm{~m}$ \\
Span & $32 \mathrm{~m}$ & $5 \mathrm{~m}$ & $2.5 \mathrm{~m}$ \\
Elastic axis & $50 \%$ chord & $50 \%$ chord & $50 \%$ chord \\
Center of gravity & $50 \%$ chord & $50 \%$ chord & $50 \%$ chord \\
Mass per unit length & $0.75 \mathrm{~kg} / \mathrm{m}$ & $0.08 \mathrm{~kg} / \mathrm{m}$ & $0.08 \mathrm{~kg} / \mathrm{m}$ \\
Moment of inertia & $0.1 \mathrm{~kg} \cdot \mathrm{m}$ & $0.01 \mathrm{~kg} \cdot \mathrm{m}$ & $0.01 \mathrm{~kg} \cdot \mathrm{m}$ \\
Torsional stiffness & $2 \times 10^{4} \mathrm{~N} \cdot \mathrm{m}^{2}$ & $\infty$ & $\infty$ \\
Bending stiffness & $4 \times 10^{4} \mathrm{~N} \cdot \mathrm{m}^{2}$ & $\infty$ & $\infty$ \\
Chordwise bending stiffness & $8 \times 10^{6} \mathrm{~N} \cdot \mathrm{m}^{2}$ & $\infty$ & $\infty$ \\
\hline \hline
\end{tabular}

\section{B. Open-loop stability}

As the flexibility of their primary structures increases, aircraft are prone to exhibit unconventional features, such as an overlap of structural and rigid-body frequencies, that is, of its aeroelastic and flight dynamic characteristics. This coupling will be investigated by evaluating the stability boundaries of the configuration introduced above (see Figure 4 and Table 1).

First of all, the aircraft is trimmed for steady level flight (in undisturbed conditions) through three inputs, namely angle of attack, AoA, elevator deflection, $\delta_{e}$, and thrust per propeller, $T$ - the rest of control surfaces, ailerons and rudder, remain undeployed. The trimming process is a tightly-coupled solution, illustrated in Figure 5. For each iteration on the trim inputs, the nonlinear static equilibrium is determined using the steady Vortex-Lattice Method (VLM) and the static beam solution. This might lead to a highly deformed 
configuration. The updated trim inputs at each new iteration are obtained by means of the standard Newton-Raphson method, ${ }^{65,66}$ and using finite differences to compute the Jacobian.

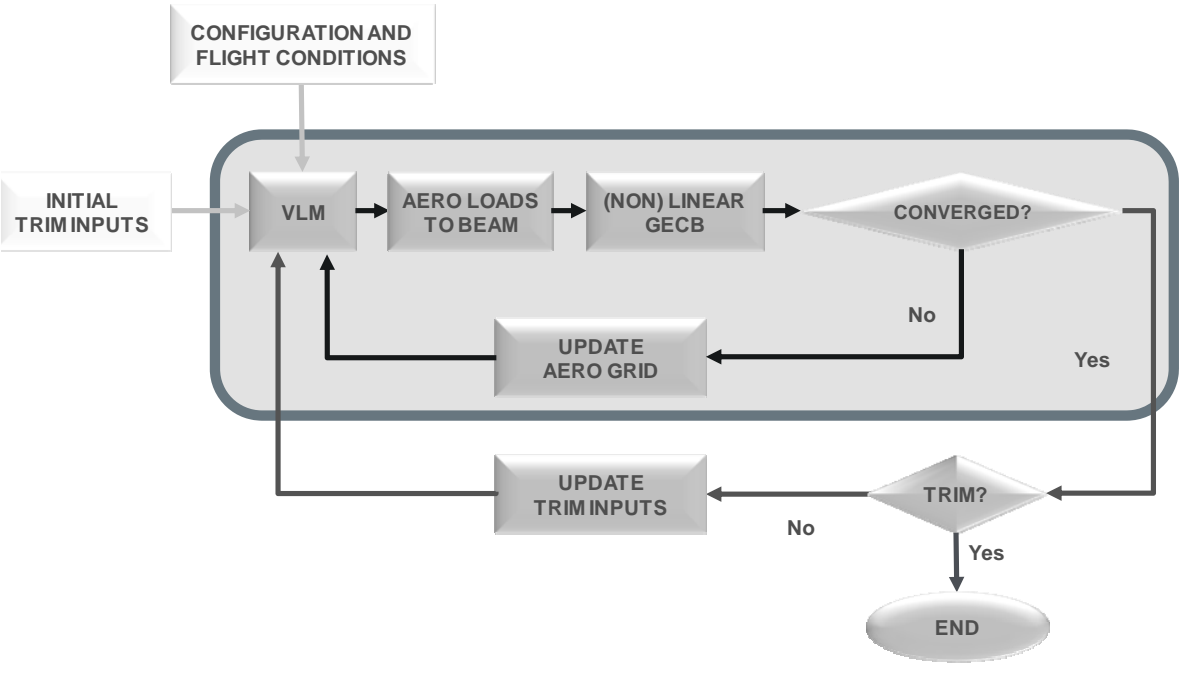

Figure 5. Tightly-coupled aircraft trimming procedure.

Once the trim conditions have been determined, a linear stability analysis is carried out about the corresponding deformed configuration. This is accomplished by solving directly the generalized eigenvalue problem obtained from by Eq. (15).

Table 2 presents the trim inputs and the stability roots. Discrete-time eigenvalues are obtained from the stability analysis, and they have been converted to the more familiar continuous-time counterparts for an easier interpretation - a positive real part of the eigenvalue indicates instability in this case. The results for the rigid aircraft are included for comparison with the fully flexible case. Note that the first few modes of the flexible vehicle will be identified by the names on their rigid-aircraft counterparts, (e.g., phugoid, short period, etc.).

Table 2. Trim and stability characteristics of rigid and flexible versions of the test aircraft. $V_{\infty}=30 \mathrm{~m} / \mathrm{s}$.

\begin{tabular}{|c|c|c|c|c|c|c|c|}
\hline \multirow[b]{3}{*}{ Rigid } & \multicolumn{3}{|c|}{ Trim inputs } & \multicolumn{4}{|c|}{ Eigenvalues } \\
\hline & AoA [deg] & $\delta_{e}[\mathrm{deg}]$ & $T[\mathrm{~N}]$ & Phugoid & Short period & Dutch roll & Spiral \\
\hline & 5.6 & -5.5 & 2.8 & $-0.011 \pm 0.27 i$ & $-4.53 \pm 1.67 i$ & $-0.41 \pm 1.30 i$ & -0.064 \\
\hline Flexible & 4.9 & -4.8 & 3.1 & $-0.0044 \pm 0.30 i$ & $-2.18 \pm 1.57 i$ & $-0.32 \pm 1.23 i$ & -0.088 \\
\hline
\end{tabular}

One might have expected a higher angle of attack to trim the flexible aircraft, since the wings deform and the lift vector is tilted inwards yielding an effective loss of vertical force. However, the twist of the wing grows rapidly with wing flexibility and provides additional lift force, because of the position of the elastic axis at mid chord. Furthermore, as the wing deforms the direction along which the thrust force acts changes significantly. Thus, equilibrium follows from several interactions and the required inputs for trim cannot be easily anticipated.

The dominant eigenvalues obtained from stability analysis, which correspond to the conventional flightdynamics modes ${ }^{67,68}$ are presented in Table 2 . Note that the stability analysis provides as many eigenvalues as states has the system, and only the most dominant ones have been included. Other flight-dynamics modes, such as the roll subsidence and yaw stiffness ${ }^{44,69}$ among others, are also an outcome of the analysis but they have been left out because they are very stable.

The role of flexibility is apparent: the real part of the two main oscillatory longitudinal modes, namely phugoid and short period, is roughly halved as the stiffness of the wing is reduced. While the effect is not dramatic and the modes remain stable, flexibility narrows down the margin. Even though the impact is smaller, the oscillatory Dutch roll and the non-oscillatory spiral, both lateral modes, are also modified. As 
it can be inferred, the spiral mode is stable in both cases thanks to the outboard dihedral - the spiral mode of the aircraft without dihedral is actually unstable ${ }^{70}$ - but wing deformations add further resilience.

The effect of flexibility is therefore twofold: on the one hand, static deformations contribute to a different stability behavior (for this particular configuration and set of conditions, the wing-tip vertical deflection is $3.22 \mathrm{~m}$ with respect to the undeformed aircraft, that is, over $20 \%$ of the wing semi-span, with an inward tip deflection of $0.28 \mathrm{~m}$ ); on the other, aeroelastic and flight-dynamics modes are coupled, and this is illustrated in Figure 6 where the flexible short-period mode is displayed. This shape has been obtained by plotting the relevant normalized eigenvectors and setting damping to zero. For visualization purposes, elastic deformations are magnified by a factor of ten with respect to the rigid-body motions.
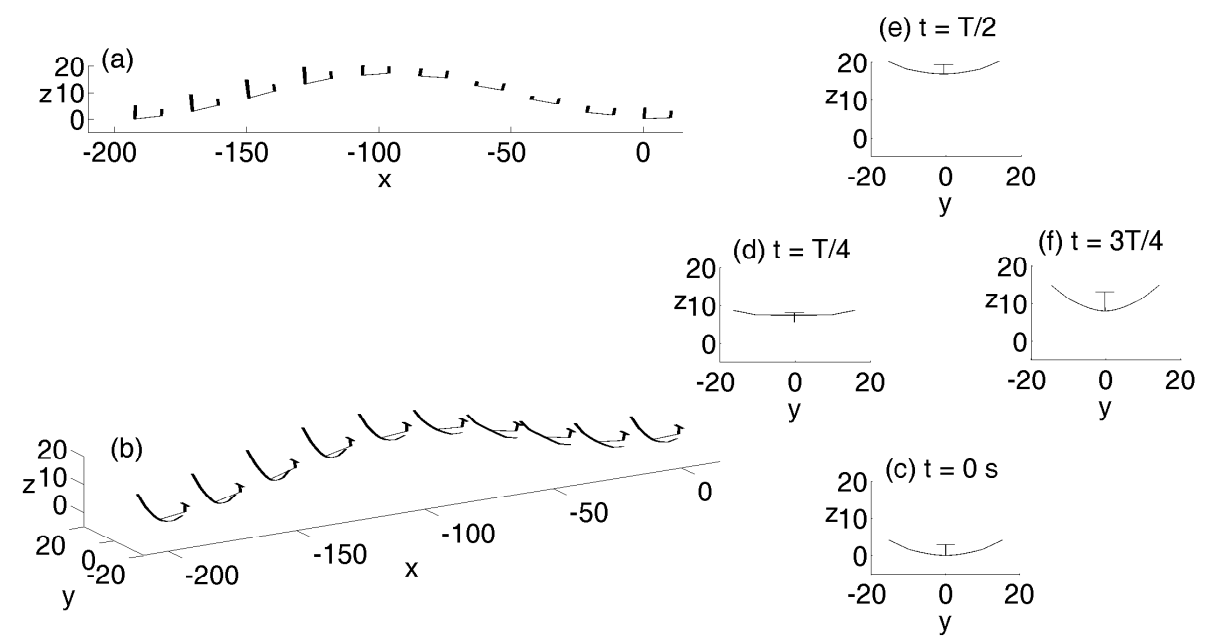

Figure 6. Flexible short-period mode with damping set to zero. One cycle, with $T=6.9$ s: (a) lateral view of the trajectory with snapshots every $\Delta t=T / 9$, (b) 3-D view with snapshots every $\Delta t=T / 9$, and (c-f) front views with snapshots every $\Delta t=T / 4$, clockwise. All dimensions $(\mathbf{x}, \mathbf{y}, \mathbf{z})$ in meters.

Figure 6(a) presents a lateral view of the trajectory followed by the aircraft, which shows the pitching and plunging motions undergone by the aircraft. The wing excursions around the static equilibrium shape can also be perceived. Figures 6(c-f) highlight the deformations of the main wing. As the aircraft pitches up and climbs first, the wings experience a downward flapping-like motion. As the vehicles pitches down and descends, the wing bends upwards, reaching the maximum tip deflections before the cycle is finished. The snapshots of the ascending and descending trajectories at the same altitude indicate the measure of the wing deflections. Figures 6(b) summarizes the above features in a 3-D view. Note that the position of the aircraft is not part of the states of the system, Eq. (16), but it is obtained by integrating body velocities.

The short period involves a change on the incidence angle, and that leads to couplings with the aerodynamic forces as the lift changes, apart from the inertial couplings with the elastic degrees of freedom. This stability mode has also been referred to as body-freedom flutter in the literature. ${ }^{71}$

To sum up, it has been shown that the aircraft is open-loop stable even for low wing stiffness. The dynamics of the vehicle as it undergoes gust-induced loads will now be investigated.

\section{Open-loop response and closed-loop load alleviation for discrete 1-cos longitudinal gusts}

As mentioned in the Introduction, HALE UAVs are especially susceptible to atmospheric turbulence and gusts. The synthesis of appropriate controllers is therefore essential to mitigate the detrimental impact on structural integrity and guidance. In this section, the response of the test aircraft defined in Figure 4 and Table 1 is studied under "1-cos" discrete longitudinal gusts.

First of all the open-loop dynamics are investigated. A spanwise-constant gust is assumed and the critical gust length is determined using as metric the root bending moment of the main wing. For this given gust, a PID controller is designed next in order to reduce the loads, using ailerons. Finally, a non-constant spanwise distribution is considered, given by the DARPA gust. ${ }^{72}$ This poses a more severe condition on the root bending moment, as will be shown by the open-loop results, and the previously designed aileron controller will again be employed for load alleviation in closed-loop. 


\section{Gust tuning}

The inclusion of gust disturbances in the standard (nonlinear) UVLM is straightforward, and the implementation of a discrete "1-cos" pulse is described, for instance, by Wang et al. ${ }^{20}$ The gust-induced velocities affect the non-penetration boundary condition at the collocation points, Eq. (1). They also modify the expression for the aerodynamic loads, Eq. (4), through the local aerodynamic velocities, $\dot{\boldsymbol{\zeta}}_{b}$. Finally, the gust will propagate into the wake if this is considered fully force-free, hence affecting the local velocities at the wake grid, $\boldsymbol{V}$ in Eq. (2), and changing its shape. Note that the assumption is that the gust profile is not modified by the vehicle.

The linear state-space formulation described in Section IV.C can also incorporate prescribed gust profiles in a straightforward way. The gust-induced velocities affect the linearized non-penetration boundary condition and aerodynamic-load equations. Recall that this linear formulation does not include a force-free wake, which is frozen at the equilibrium conditions.

Stationary, discrete "1-cos" pulses, constant spanwise, are considered first. The gust-induced velocity only has a vertical component. At a penetration length through the disturbance, $x_{g}(t)$, the gust velocity is given by

$$
V_{g}(t)=\frac{1}{2} V_{g 0}\left[1-\cos \left(\frac{2 \pi x_{g}}{L_{g}}\right)\right], \quad 0 \leq x_{g} \leq L_{g},
$$

where $V_{g 0}$ is the gust intensity, and $L_{g}$ its length (gradient). At time step $n$, the column vector with the gust velocities at all collocation points, $\boldsymbol{V}_{g}^{n}$, coincides with $\Delta \boldsymbol{u}_{d}^{n}$ in Eq. (17), where the relationship between the penetration length and time is given by

$$
x_{g}=V_{\infty} t^{n}-x_{k},
$$

and where $x_{k}$ is the coordinate of the $k^{\text {th }}$ collocation point on the body-fixed frame, assuming the origin of this frame is located at the edge of the incident gust at the beginning of the motion.

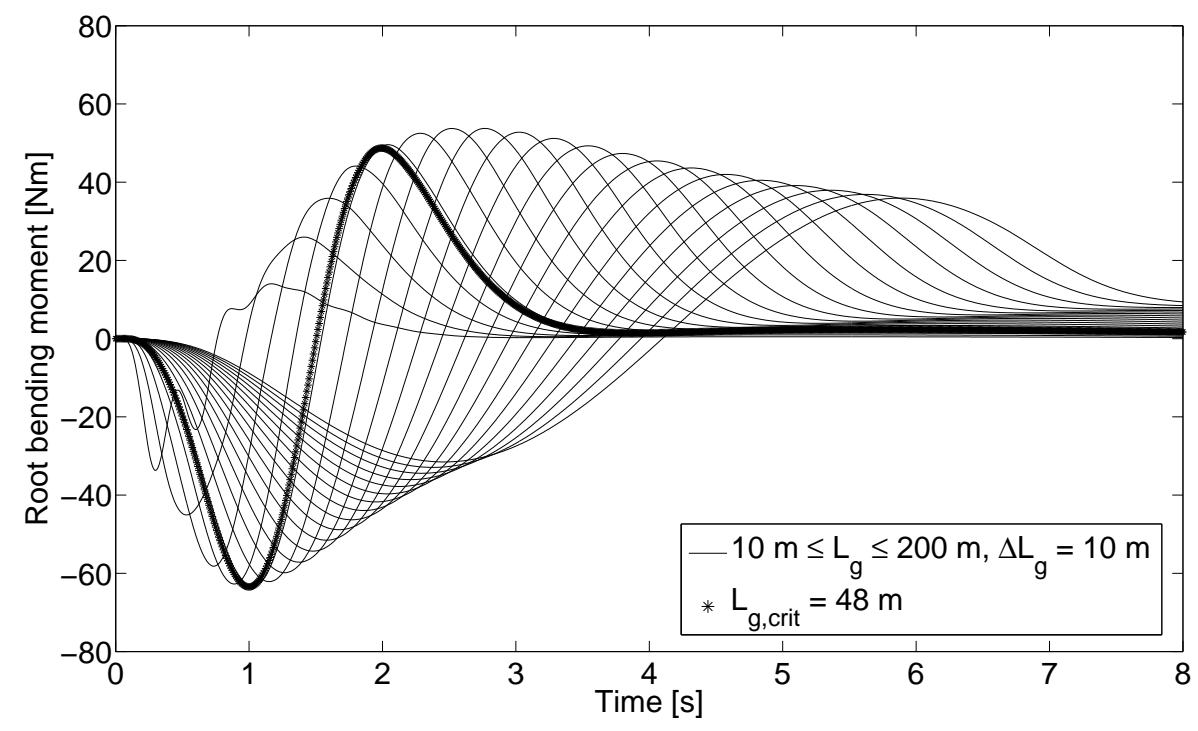

Figure 7. Variation in the root bending moment of the main wing of the test aircraft undergoing "1-cos" gust disturbances of different lengths in open-loop. Critical gust length is $L_{g, \text { crit }}=48 \mathbf{m}$. $V_{g 0}=0.01 V_{\infty}$, with $V_{\infty}=30$ $\mathrm{m} / \mathrm{s}$.

In order to determine the critical gust length, the root bending moment is computed through the openloop linear state-space equations. A small gust intensity of $V_{g 0}=0.01 V_{\infty}$ is simulated, so that the results remain linear. Figure 7 presents results for gusts of different lengths. Gust lengths from $10 \mathrm{~m}$ to $200 \mathrm{~m}$ have been evaluated, in increments of $10 \mathrm{~m}$, and after refining to increments of $1 \mathrm{~m}$, the critical gust length is found to be $L_{g, \text { crit }}=48 \mathrm{~m}$, or 48 chord-lengths. The figure presents variations of the root bending moment about the static equilibrium value, $M_{B 0}=-1865 \mathrm{Nm}$. This value corresponds to the deformed wing of the trimmed vehicle, which exhibits deflections of $20 \%$ of the semi-span. The plots illustrate the typical trend 
for a flexible member due to a gust: the wing experiences up-bending motions as the gust velocity increases, and then bends downwards as the gust has been left behind. Note that due to the initial static deformations, a positive (upwards) gust constitutes the most restrictive case.

\section{Load alleviation through PID control}

As a next step, the possibility of alleviating the maximum stresses is explored. This will be achieved via PID control $^{73}$ actuating the outboard ailerons of the main wing - the actuation mechanisms are not included in this model. Aerodynamic control surfaces are directly modeled in the nonlinear UVLM by prescribed deflections of trailing edge panels and the corresponding rotation of normal vectors. In the linear case, under the assumption of a frozen aerodynamic geometry, only the rotation of the normal vectors is included, but the relative position between panels remains constant. In addition, whereas the nonlinear version of the equations caters for the velocities incurred by the motions of control surfaces, the linear state-space formulation does not capture them, and is therefore a quasi-steady approximation of the deployment.

The above "1-cos" gust is considered, for the critical length of $L_{g, c r i t}=48 \mathrm{~m}$ and with a gust intensity of $V_{g 0}=0.01 V_{\infty}$. Using the linearized equations, a PID controller acting on the ailerons located at the dihedral members is designed in order to reduce wing-root bending moments. Figure 8 compares the openand closed-loop responses. The moments at which the maximum gust velocity hits the main wing, $t_{1}$, and the empennage, $t_{2}$, are indicated. All plotted variables correspond to variations about equilibrium conditions, which are non-zero for the root bending moment and the vertical wing-tip deflection.
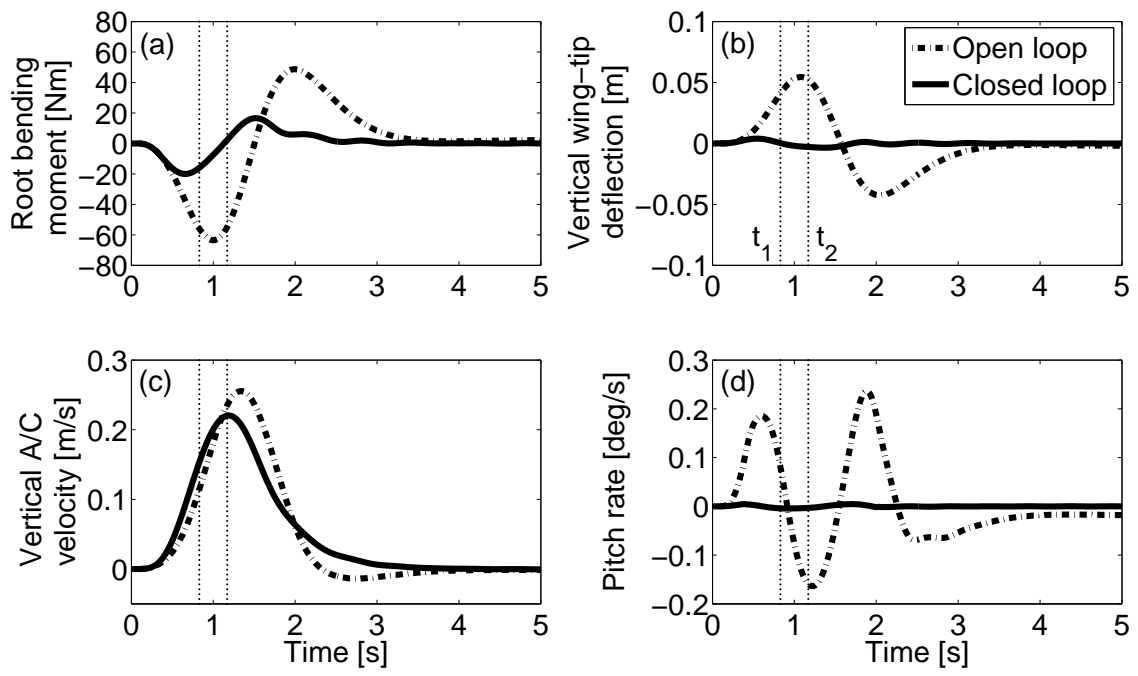

Figure 8. Closed-loop load alleviation under a "1-cos" spanwise constant gust of maximum intensity $V_{g 0}=$ $0.01 V_{\infty}$, using PID control for the ailerons. Variations in: (a) root bending moment, (b) vertical wing-tip deflection, (c) vertical aircraft velocity, and (d) pitch rate.

There are different approaches to automatically tune the gains of a PID controller, ${ }^{74}$ being the ZieglerNichols method ${ }^{75}$ probably the most popular one. After some trial and error, it has been found that direct proportional gain is good enough, and it has been set to $k_{p}=1 \times 10^{-3} \mathrm{deg} /(\mathrm{Nm})$. That is, the command for aileron deflection, $\delta_{a}$, is given by $\delta_{a}=1 \times 10^{-3} M_{B}$.

The benefits of introducing aileron control are manifest. There is a $68 \%$ reduction in the maximum variation experienced by the root bending moment, i.e., on the maximum oscillation about the trimmed conditions. The position of the wing tip remains nearly constant, and so does the pitch rate. Hence, interestingly, the aileron deployment also moderates body motions, and even the vertical velocity of the aircraft decreases - note that body-velocities are measured at the root of the main wing. In open-loop, as the gust reaches the front of the aircraft the main wing bends upwards, but the nose is also lifted with respect to the tail, inducing a pitch rate. The ailerons, which are driven to reduce wing strains, reduce the lift of the wing to counteract the gust. Apart from the effect on wing deflections, this also creates a nose-down pitching moment that balances the gust-induced pitch rate.

Other control techniques have also been considered for gust-load alleviation in similar platforms. ${ }^{22,76} \mathrm{In}$ these works the root bending moment was reduced by about $10 \%$ by implementing robust $\mathcal{H}_{\infty}$ control. This 
proves that the PID controllers used here can bestow a significantly higher impact on the variables to be regulated. However, this has been achieved assuming "1-cos", spanwise symmetric gusts, which constitutes an idealization of the random atmospheric disturbances found in reality. In these scenarios, robust control provides a more general approach.

In practice, constraints would need to be enforced on maximum control-surface deflections and deployment rates. This is analyzed next for a more restrictive case.

\section{Load alleviation on a gust of sinusoidal spanwise distribution}

In the context of the Vulture II program, DARPA furnished some guidelines for discrete load criteria. ${ }^{72}$ Apart from the uniform "1-cos" pulses already studied, a non-uniform gust of spanwise symmetrical sinewake shape is proposed. In this case, assuming again a "1-cos" form longitudinally, the gust velocity at a position $\left(x_{g}(t), y_{g}(t)\right)$ within the gust is given by

$$
V_{g}(t)=-\frac{1}{2} V_{g 0}\left[1-\cos \left(\frac{2 \pi x_{g}}{L_{g}}\right)\right] \cos \left(\frac{2 \pi y_{g}}{\lambda_{g}}\right), \quad 0 \leq x_{g} \leq L_{g}, \quad-\frac{\lambda_{g}}{2} \leq y_{g} \leq \frac{\lambda_{g}}{2},
$$

where $\lambda_{g}$ is the spanwise wavelength of the gust. This equation is an extension of Eq. (18), introducing a correcting factor to account for the spanwise location of the point within the gust, given by $y_{g}$. In the present implementation, as the spanwise gust distribution is assumed symmetric, $y_{g}$ coincides with the spanwise coordinate of the collocation points on the body-fixed frame.

The DARPA report suggests a tuning process for the gust wavelength, adjusting the gust intensity accordingly. In this work, in order to maintain linearity, a small gust intensity of $V_{g 0}=0.01 V_{\infty}$ is considered again. For a fixed intensity, the most challenging wavelength would be the one that matches the wing span, since it minimizes the alleviation provided by the aircraft plunging motion: as the wing tips bend upwards, an opposite gust velocity of the same magnitude pulls the fuselage nose downwards, hence maximizing bending strains. As a result, for the subsequent results, it has been chosen $\lambda_{g}=32 \mathrm{~m}$.

Figure 9 compares the open- and closed-loop responses for the test aircraft under this gust. The vertical body-velocity, measured at the wing root, is negative when the aircraft encounters the gust, and the impact of this can be seen in the maximum root bending moment, which is larger (in absolute value) than for the spanwise constant gust (Figure 8). The advantages of introducing aileron deployment are again evident, bringing down the peak values in all metrics.
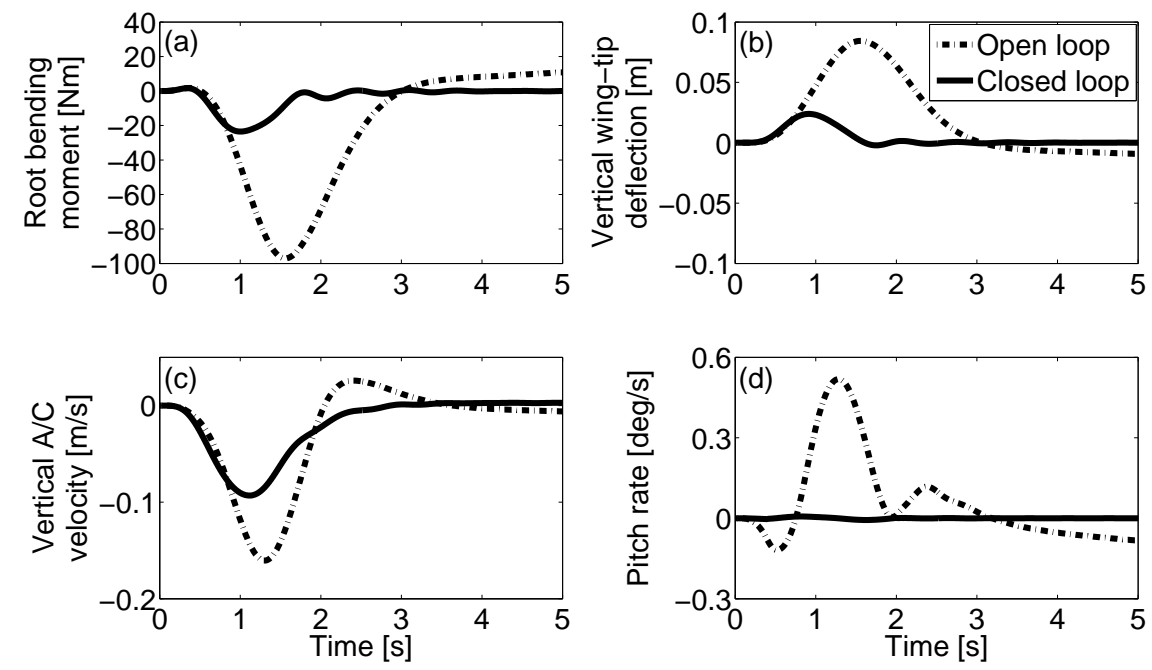

Figure 9. Closed-loop load alleviation under a "1-cos" spanwise sinusoidal gust of maximum intensity $V_{g 0}=$ $0.01 V_{\infty}$, using PID control for the ailerons. Variations in: (a) root bending moment, (b) wing-tip deflection, (c) vertical velocity, and (d) pitch rate.

The closed-loop results in Figure 9 have been obtained with the same proportional aileron controller used for the spanwise constant gust, that is, with a gain of $k_{p}=1 \times 10^{-3} \mathrm{rad} /(\mathrm{Nm})$. In order to assess the feasibility of this controller, Figure 10 presents the commanded actuation, together with the velocities experienced by selected points of the aircraft due to the disturbance. 

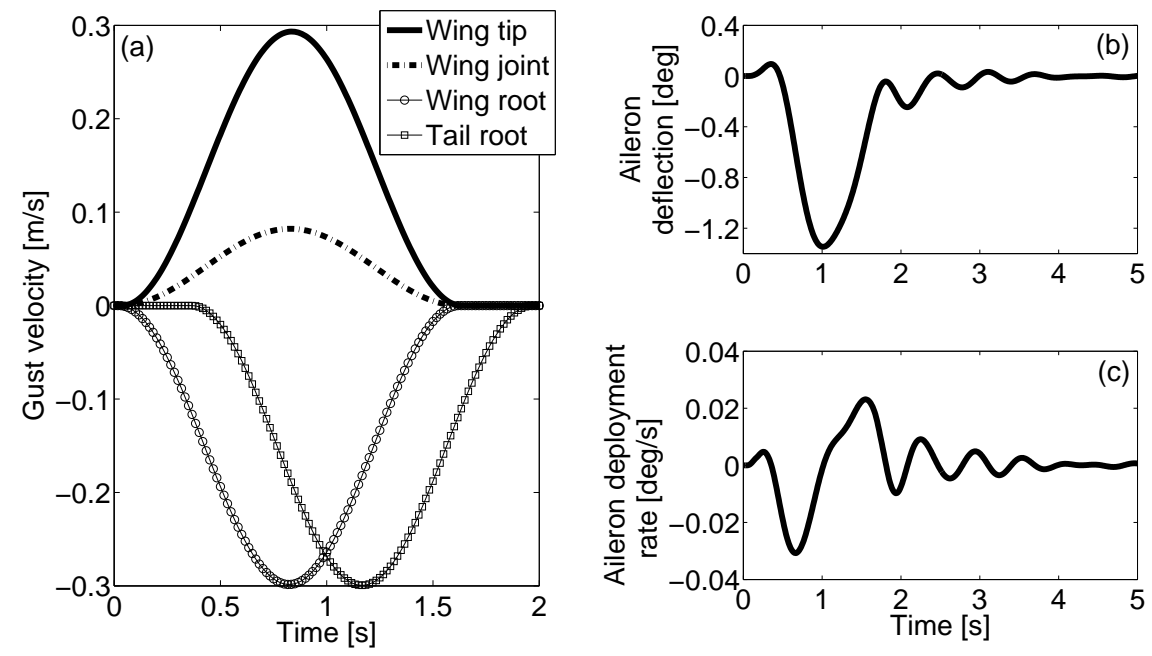

Figure 10. Gust and control inputs for the closed-loop response under a "1-cos" spanwise sinusoidal gust of maximum intensity $V_{g 0}=0.01 V_{\infty}$ : (a) gust velocities at selected locations on the aircraft, (b) commanded aileron deflections, and (c) commanded aileron deployment-rates.

Figure 10(a) plots the gust velocities at the wing tip, at the linkage between the two members the main wing consists of, at the wing root, and at the tail root. The non-uniform spanwise distribution can be observed. The wing-tip panel reaches its maximum velocity slightly later that the root, due to the chordwise deformations.

Figure 10(b) depicts the deflections commanded to the aileron, and Figure 10(c) the deployment rate, which has be obtained from the aileron deflections using a first-order backward differentiation - recall that the linearized model does not account for control-surface velocities. For this small intensity gust, both magnitudes remain fairly small. It is worth pointing out that, as this analysis is linear, an increase in the gust intensity would lead to an linear increase of aileron commands. Hence, even for a substantially stronger gust, say $V_{g 0}=0.1 V_{\infty}$, the maximum aileron deflection would be $\delta_{a}=-13.5 \mathrm{deg}$. However, a higher intensity gust might lead to geometrically nonlinear deformations, large rigid-body motions, or both, which would not be accurately captured by the linear approximation. The saturation threshold of the controller might also be reached in these cases. These effects will be explored in the next section.

\section{Open-loop response and closed-loop trajectory control for a non-uniform thermal distri- bution}

To conclude the numerical results of this paper, the non-uniform thermal distribution proposed by Su and Cesnik $^{13}$ will be considered. In that work, a time- and space- dependent gust was assumed, with the vertical velocity component given by

$$
V_{g}(r, \beta, t)=\frac{1}{2} V_{g 0}\left[1-\cos \left(\frac{2 \pi t}{t_{g}}\right)\right] \sqrt{\left(A_{E} \cos \beta\right)^{2}+\left(A_{N} \sin \beta\right)^{2}},
$$

with

$$
\begin{aligned}
& A_{E}(r)=\sin \left\{\frac{\pi}{2}\left[1-\left(\frac{r}{r_{g}}\right)^{n_{E}}\right]\right\} \\
& A_{N}(r)=\sin \left\{\frac{\pi}{2}\left[1-\left(\frac{r}{r_{g}}\right)^{n_{N}}\right]\right\}, \quad 0 \leq r \leq r_{g},
\end{aligned}
$$

and where $E$ and $N$ stand for, respectively, East and North. $r$ the distance from a point to the gust center, $\beta$ is the angle of the point/gust-center line with respect to the East direction, and $t$ is the time elapsed since the beginning of the gust, being $t_{g}$ the gust duration. This is a circular distribution on the plane, with gust radius $r_{g}$. The maximum velocity occurs at the center point, and it vanishes to zero at the boundaries. 
Obviously, a wide range of parameters can be chosen for the gust description. In this work, as in the original reference, $n_{E}=1$ and $n_{N}=2$ will be assumed. A gust radius of $r_{g}=20 \mathrm{~m}$ is considered, and its center is aligned with the wing tip in the forward flight direction. This will create asymmetric loads on the aircraft: whereas the starboard wing will be fully hit by the gust, only the region close to the root of the port wing will see the disturbance. The gust duration is chosen to be $t_{g}=2.5 \frac{r_{g}}{V_{\infty}}$, which implies that the maximum temporal gust velocities will be reached when the aircraft has advanced $25 \mathrm{~m}$ within the guts, assuming it was initially located at the boundary of the thermal.

In this test case, the endeavors will focus on guiding the aircraft through the gust as smoothly as possible, without any explicit attempt on reducing strains. To that goal, the main-wing ailerons will provide roll control, the elevator will be employed for pitch regulation, and the rudder will steer the vehicle reducing yawing motions.

Figure 11 compares the open- and closed-loop responses for the thermal distribution given by Eqs. (2122 ). The variation in root bending moment of the main wing is displayed, along with variations in aircraft position and Euler angles. It can be observed that the aircraft follows a divergent trajectory in open loop. In contrast, the PID controllers successfully drive the aircraft through the disturbance, significantly reducing the departure from the original flight path and efficiently bringing down to zero the variations in orientation. Recall that, for the port wing, the disturbance only affects the region very close to the root (aircraft centerline), and even here the velocities are very small compared to those experienced by the starboard wing. This creates a rolling moment on the aircraft, which is counteracted by the ailerons in the closed-loop quite efficiently. On the other hand, note that the penalty on the maximum root bending moment by the implementation of these control laws is negligible.
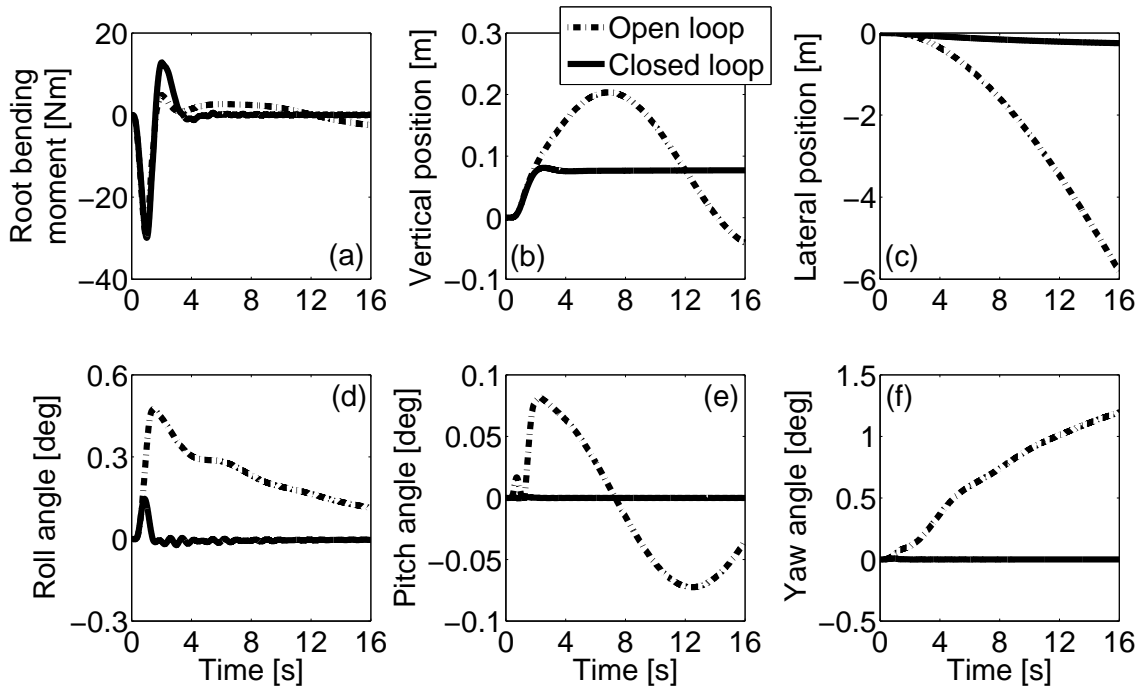

Figure 11. Closed-loop trajectory control under a non-uniform thermal of maximum intensity $V_{g 0}=0.01 V_{\infty}$, using PID control for the ailerons, elevator, and rudder. Variations in: (a) root bending moment, (b) aircraft vertical position, (c) aircraft lateral position, (d) roll angle, (e) pitch angle, and (f) yaw angle.

Table 3 presents the chosen gains in order to achieve the results in Figure 11. Ailerons, elevator, and rudder PID controllers respectively actuate upon roll, pitch, and yaw rates. Ailerons on the starboard and port wings receive the same signal but of opposite sign. The absolute value is given for all gains, since the signs depend on the axes convention, which differs across disciplines.

Table 3. PID control gains for trajectory regulation.

\begin{tabular}{lccc}
\hline \hline & $k_{p}[\operatorname{deg} /(\operatorname{deg} / \mathrm{s})]$ & $k_{i}[(\operatorname{deg} /(\operatorname{deg} / \mathrm{s})) / \mathrm{s}]$ & $k_{d}[(\operatorname{deg} /(\operatorname{deg} / \mathrm{s})) \cdot \mathrm{s}]$ \\
\hline Starboard aileron & 0.2 & 15 & 0.01 \\
Elevator & 3.0 & 300 & 0.02 \\
Rudder & 1.0 & 100 & 0.00 \\
\hline \hline
\end{tabular}


Figure 12 shows disturbance and control inputs into the system. Figure 12(a) presents gust velocities at selected locations, which enable the spanwise distribution on the starboard wing to be visualized, along with the longitudinal propagation from wing to empennage - recall that the gust is stationary, and this "propagation" is due to the aircraft forward motion. The gains of Table 3 yield the control-surface commands displayed in Figures 12(b), (c) and (d).
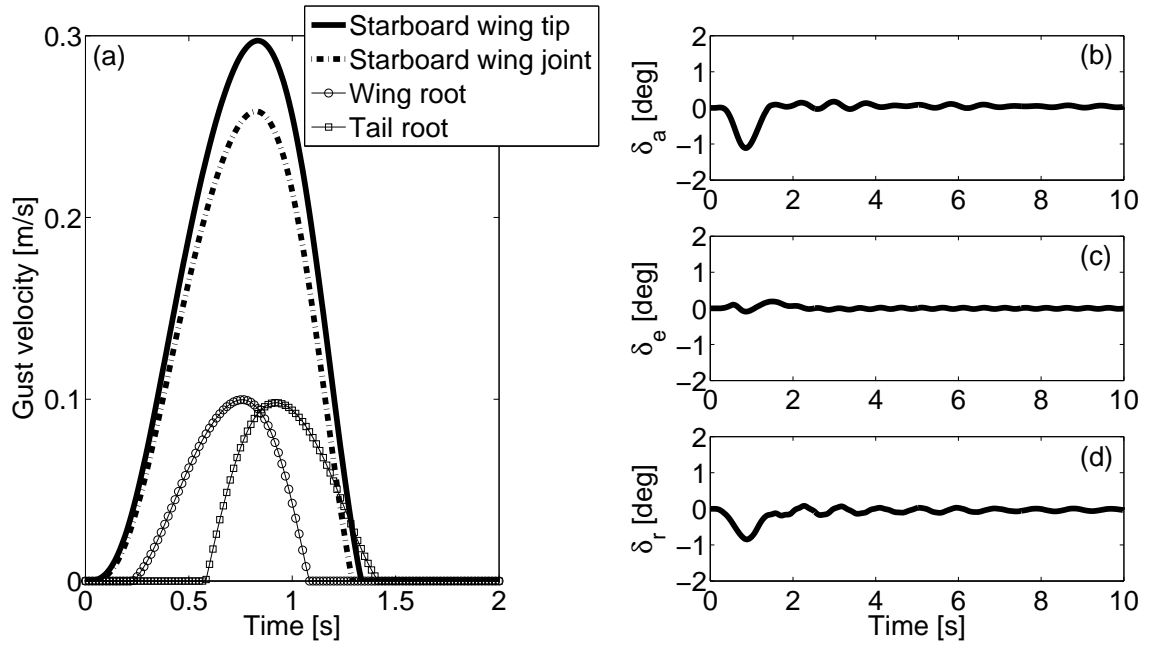

Figure 12. Gust and control inputs for the closed-loop response under a non-uniform thermal of maximum gust intensity $V_{g 0}=0.01 V_{\infty}$ : (a) gust velocities at selected locations on the aircraft, (b) commanded starboard aileron deflections, (c) commanded elevator deflections (with respect to the trim value), and (d) commanded rudder deflections.

For this small intensity gust, $V_{g 0}=0.01 V_{\infty}$, the control-surface deflections presented in Figure 12 are attainable. However, very large gust intensities would most probably lead to controller saturation. Figure 13 compares temporal response and actuation requirements for increasing gust intensities. The variations in tip displacement of the main wing, lateral position of the aircraft (measured at the nose), and aileron commands are plotted for three values of $V_{g 0}$. The linearized model has been used.
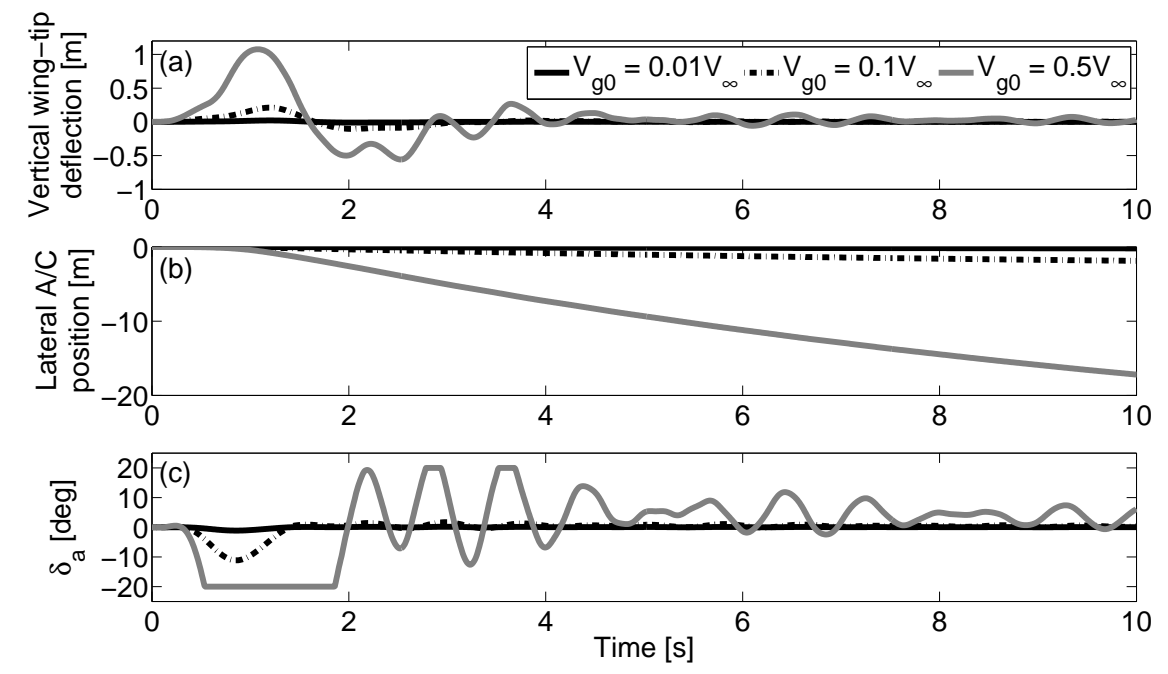

Figure 13. Closed-loop response under a non-uniform thermal at various gust intensities: (a) $V_{g 0}=0.01 V_{\infty}$, (b) $V_{g 0}=0.1 V_{\infty}$, and (c) $V_{g 0}=0.5 V_{\infty}$.

The controller performs successfully for the small and medium intensities, but it is obviously not suitable for the large value of $V_{g 0}=0.5 V_{\infty}$. In this case, ailerons and rudder (the latter not included in the figure) reach the saturation limit, which has been defined to be 20 deg control-surface deflection. Even though structural deformations and rigid-body motions remain linear, the controller is not effective and the path 
followed by the aircraft deviates from the commanded trajectory. In this situation, the assumption of frozen aerodynamic geometry to linearize the aerodynamic equations might no longer be appropriate, since the relative position between lifting surfaces and wakes changes significantly. In any case, the effective angles of incidence under this gust are very large and will lead to flow separation.

To conclude, the open- and closed-loop aircraft trajectories are compared in Figure 14, for the thermal distribution with $V_{g 0}=0.1 V_{\infty}$. Figure 14(a) shows a top view, including the gust region, and Figure 14(b) a 3-D view. The excellent authority of the PID controllers can be clearly appreciated. Whereas the openloop path presents a large departure, the closed-loop response closely follows the intended flight trajectory. Besides, the dynamics remain linear with small elastic deformations, rigid-body motions, and control signals, rendering valid the linear approximation. Thus the controller accomplishes what it was designed for, and proves the capabilities of the approach for this relatively strong gust.

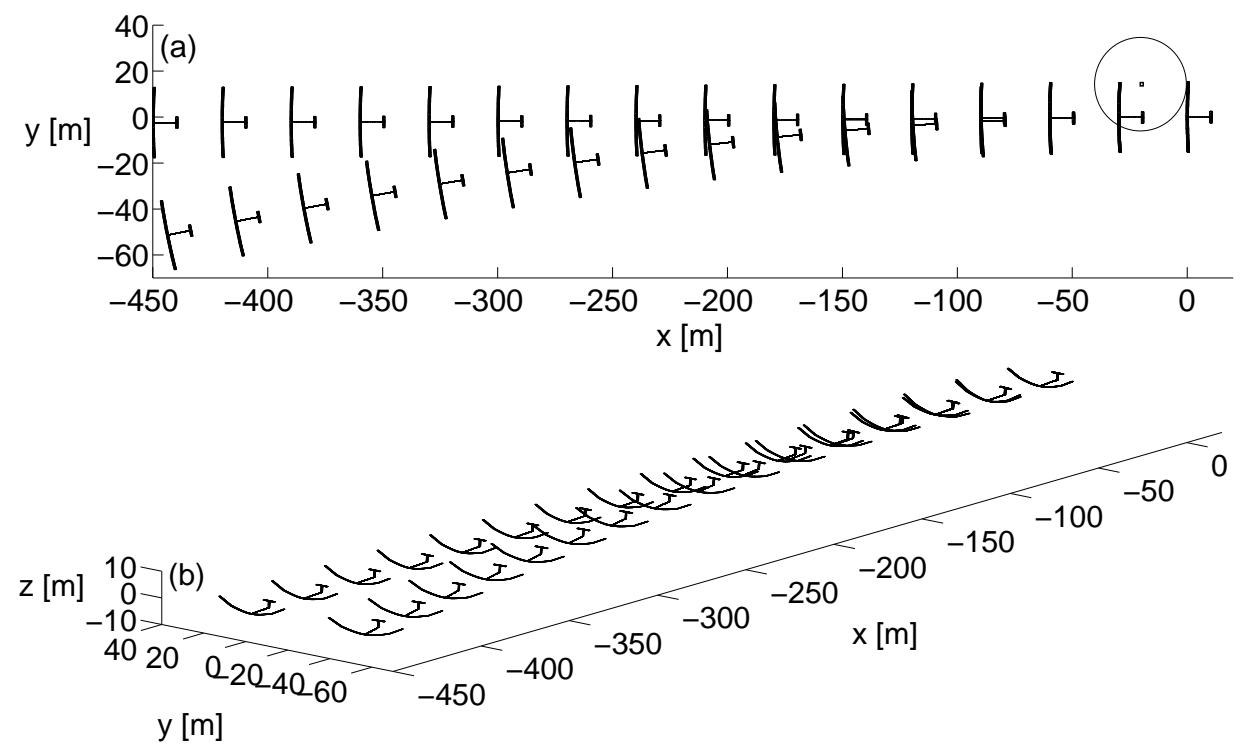

Figure 14. Open- and closed-loop aircraft trajectories for $V_{g 0}=0.1 V_{\infty}$, with snapshots every $V_{\infty} \Delta t / r_{g}=1.5$ : (a) top view indicating gust region, and (b) 3-D view.

\section{Conclusions}

A multidisciplinary, medium-fidelity model has been presented for the simulation of the dynamics of lowspeed flexible aircraft, and its capabilities have been illustrated by analyzing open-loop stability and closedloop gust alleviation on a representative HALE platform. In order to accomplish this, the aerodynamics are described by the Unsteady Vortex-Lattice Method, which is coupled with a Geometrically-Exact Composite Beam model that encompasses elastic and rigid-body degrees of freedom. The flexible-body equations are discretized using a Newmark- $\beta$ method, and the mapping is carried out assuming that airfoils (wing crosssections) remain rigid.

In its general nonlinear form, the framework can capture the instantaneous shape of the lifting surfaces and the free wake, including large geometrically-nonlinear deflections, in-plane motions, and 3-D aerodynamics interference effects. In turn, the linearization of the equations leads to a seamlessly integrated discretetime state-space system. This is achieved by performing small perturbation analyses about an equilibrium configuration, which can exhibit large static deformations, and assuming a frozen aerodynamic geometry. The resulting monolithic assembly offers increased fidelity compared to traditional tools in aircraft aeroelasticity and flight dynamics, such as strip theory or the Doublet-Lattice Method, and provides a powerful and computationally-efficient tool: for stability analysis, through a direct eigenvalue problem; and for open- and closed-loop dynamic response, by expanding the homogeneous problem to account for exogenous disturbances and control inputs.

The numerical results have exploited some of the capabilities of this linearized model. First of all, a linear stability analysis has been performed on a flexible aerial platform, exposing the coupled aeroelastic 
and flight-dynamic features of the vehicle. It has been shown that, for this particular aircraft, the effect of flexibility is visible but not dramatic.

Next, the paper has delved into gust response, addressing both load alleviation and trajectory regulation using PID control. Various types of discrete models of atmospheric disturbances have been considered, including longitudinal symmetric gusts and asymmetric thermal distributions. For the test case analyzed, simple PID has been shown to perform well in all cases, substantially bringing down bending strains (over two-thirds reduction!) and providing the necessary guidance authority.

Exogenous disturbances have been represented by discrete, deterministic models. Therefore, the benefits furnished by the closed-loop controllers can only be seen as indicative of what could be achieved for these idealized disturbances. Atmospheric turbulence and gusts are stochastic phenomena, and robust control synthesis ${ }^{77}$ should be investigated next, whereby random distributions are catered for. The linear monolithic framework presented in this paper is ideally suited for the implementation of these techniques, as well as for the straightforward generation of the reduced order models that might be required as a previous step. In addition, this formulation is expected to constitute a fast and valuable tool to address multidisciplinary optimization and uncertainty modeling in low-speed vehicle design.

\section{Acknowledgments}

Joseba Murua gratefully acknowledges the financial support provided by the Department of Education, Universities and Research of the Basque Government.

\section{References}

\footnotetext{
${ }^{1}$ Noll, T. E., Ishmael, S. D., Henwood, B., Perez-Davis, M. E., Tiffany, G. C., Gaier, M., Brown, J. M., and Wierzbanowski, T., "Technical Findings, Lessons Learned, and Recommendations Resulting from the Helios Prototype Vehicle Mishap," NATO/RTO AVT-145 Workshop on Design Concepts, Processes and Criteria for UAV Structural Integrity, Florence, Italy, May 2007.

${ }^{2}$ Hoblit, F. M., Gust Loads on Aircraft: Concepts and Applications, AIAA Education Series, American Institute of Aeronautics and Astronautics, Inc., 1988.

${ }^{3}$ Lomax, T. L., Structural Loads Analysis for Commercial Transport Aircraft: Theory and Practice, AIAA Education Series, American Institute of Aeronautics and Astronautics, Inc., 1996.

${ }^{4}$ Howe, D., Aircraft Loading and Structural Layout, Aerospace Series, Professional Engineering Publishing, 2004.

${ }^{5}$ Wright, J. R. and Cooper, J. E., Introduction to Aircraft Aeroelasticity and Loads, John Wiley \& Sons Ltd, 2007.

6 "Certification Specifications, European Aviation Safety Agency," .

7 "Federal Aviation Administration Regulations, Part 25 Airworthiness Standards: Transport Category Airplanes. U. S. Department of Transportation," .

${ }^{8}$ Zole, A. and Karpel, M., "Continuous Gust Response and Sensitivy Derivatives Using State-Space Models," Journal of Aircraft, Vol. 31, No. 5, September-October 1994, pp. 1212-1214.

${ }^{9}$ Liepmann, H. W., "On the application of statistical concepts to the buffeting problem," Journal of Aeronautical Sciences, Vol. 19, No. 12, 1952, pp. 793-800.

${ }^{10}$ Diederich, F. W. and Drischler, J. A., "Effect of spanwise variations in gust intensity on the lift due to atmospheric turbulence," NACA TN 3920, April 1957.

${ }^{11}$ Houbolt, J. C., Steiner, R., and Pratt, K. G., "Dynamic response of airplanes to atmospheric turbulence including flight data on input and response," NASA TR R-199 (initially presented as an AGARD paper, July 1962), June 1964.

${ }^{12}$ Campbell, C. W., "Monte Carlo Turbulence Simulation Using Rational Approximations to von Kármán Spectra," AIAA Journal, Vol. 24, No. 1, 1986, pp. 62-66.

${ }^{13} \mathrm{Su}$, W. and Cesnik, C. E. S., "Dynamic Response of Highly Flexible Flying Wings," AIAA Journal, Vol. 49, No. 2, February 2011, pp. 324-339.

${ }^{14}$ Albano, E. and Rodden, W. P., "A Doublet-Lattice Method for Calculating Lift Distributions on Oscillating Surfaces in Subsonic Flow," AIAA Journal, Vol. 7, No. 2, 1969, pp. 279-285.

${ }^{15}$ Roger, K. L., "Airplane Math Modelling and Active Aeroelastic Control Design," Tech. rep., AGARD-CP-228, 1977.

${ }^{16}$ Eversman, W. and Tewari, A., "Consistent rational fraction approximation for unsteady aerodynamics," Journal of Aircraft, Vol. 28, No. 9, September 1991, pp. 545-552.

${ }^{17}$ Karpel, M., "Design for Active Flutter Suppression and Gust Alleviation Using State Space Aeroelastic Modeling," Journal of Aircraft, Vol. 19, No. 3, 1982, pp. 221-227.

${ }^{18}$ Raveh, D. E., "Gust-Response Analysis of Free Elastic Aircraft in the Transonic Flight Regime," Journal of Aircraft, Vol. 48, No. 4, July-August 2011, pp. 1204-1211.

${ }^{19}$ Patil, M. J. and Hodges, D. H., "Flight Dynamics of Highly Flexible Flying Wings," Journal of Aircraft, Vol. 43, No. 6, November-December 2006, pp. 1790-1798.

${ }^{20}$ Wang, Z., Chen, P. C., Liu, D. D., and Mook, D. T., "Nonlinear-Aerodynamics/Nonlinear-Structure Interaction Methodology for a High-Altitude Long-Endurance Wing," Journal of Aircraft, Vol. 47, No. 2, 2010, pp. 556-566.
} 
${ }^{21}$ Wildschek, A., Stroscher, F., Klimmek, T., Šika, Z., Vampola, T., Valášek, M., Gangsaas, D., Aversa, N., and Berard, A., "Gust load alleviation on a large blended wing body airliner," $2^{\gamma^{t h}}$ International Congress of the Aeronautical Sciences, Nice, France, 2010.

${ }^{22}$ Cook, R., Palacios, R., and Goulart, P., "Robust Gust Alleviation and Stabilization of Very Flexible Aircraft," AIAA Journal, 2012, [Under review].

${ }^{23}$ Maute, K., Nikbay, M., and Farhat, C., "Coupled Analytical Sensitivity Analysis and Optimization of Three-Dimensional Nonlinear Aeroelastic Systems," AIAA Journal, Vol. 39, No. 11, 2001, pp. 2051-2061.

${ }^{24}$ Geuzaine, P., Brown, G., Harris, C., and Farhat, C., "Aeroelastic Dynamic Analysis of a Full F-16 Configuration for Various Flight Conditions," AIAA Journal, Vol. 41, No. 3, 2003, pp. 363-371.

${ }^{25}$ Garcia, J. A., "Numerical Investigation of Nonlinear Aeroelastic Effects on Flexible High-Aspect-Ratio Wings," Journal of Aircraft, Vol. 42, No. 4, July-August 2005, pp. 1025-1036.

${ }^{26}$ van Schoor, M. C. and van Flotow, A. H., "Aeroelastic Characteristics of a Highly Flexible Aircraft," Journal of Aircraft, Vol. 27, No. 10, October 1990, pp. 901-908.

${ }^{27}$ Drela, M., "Integrated Simulation Model for Preliminary Aerodynamic, Structural, and Control-Law Design of Aircraft," $40^{\text {th }}$ AIAA Structures, Structural Dynamics and Materials Conference, AIAA 99-1934, St. Louis, MO, USA, April 1999, pp. $1644-1656$.

${ }^{28}$ Patil, M. J., Hodges, D. H., and Cesnik, C. E. S., "Nonlinear Aeroelasticity and Flight Dynamics of High-Altitude Long-Endurance Aircraft," Journal of Aircraft, Vol. 38, No. 1, 2001, pp. 88-94.

${ }^{29}$ Meirovitch, L. and Tuzcu, I., "Unified Theory for the Dynamics and Control of Maneuvering Flexible Aircraft," AIAA Journal, Vol. 42, No. 4, April 2004, pp. 714-727.

${ }^{30}$ Shearer, C. M. and Cesnik, C. E. S., "Nonlinear Flight Dynamics of Very Flexible Aircraft," Journal of Aircraft, Vol. 44, No. 5, September-October 2007, pp. 1528-1545.

${ }^{31}$ Palacios, R., Cesnik, C. E. S., and Reichenbach, E. Y., "Re-examined Structural Design Procedures for Very Flexible Aircraft," International Forum on Aeroelasticity and Structural Dynamics, Stockholm, Sweden, July 2007.

${ }^{32}$ Palacios, R., Murua, J., and Cook, R., "Structural and Aerodynamic Models in the Nonlinear Flight Dynamics of Very Flexible Aircraft," AIAA Journal, Vol. 48, No. 11, November 2010, pp. 2648-2559.

${ }^{33}$ Murua, J., Palacios, R., and Graham, J., "Modeling of Nonlinear Flexible Aircraft Dynamics Including Free-Wake Effects," AIAA Atmospheric Flight Mechanics Conference, AIAA 2010-8226, Toronto, ON, Canada, August 2010.

${ }^{34}$ Murua, J., Hesse, H., Palacios, R., and Graham, J. M. R., "Stability and Open-Loop Dynamics of Very Flexible Aircraft Including Free-Wake Effects," 52 ${ }^{\text {nd }}$ AIAA/ASME/ASCE/AHS/ASC Structures, Structural Dynamics, and Materials Conference, AIAA 2011-1915, Denver, CO, USA, April 2011.

${ }^{35}$ Murua, J., Palacios, R., and Graham, J. M. R., "Assessment of Wake-Tail Interference Effects on the Dynamics of Flexible Aircraft," AIAA Journal, 2012, [Accepted for publication].

${ }^{36}$ Katz, J. and Plotkin, A., Low-Speed Aerodynamics, Cambridge Aerospace Series, Cambridge University Press, 2nd ed., 2001.

${ }^{37}$ Hall, K. C., "Eigenanalysis of Unsteady Flows About Airfoils, Cascades, and Wings," AIAA Journal, Vol. 32, No. 12, December 1994, pp. 2426-2432.

${ }^{38}$ Dovgiǐ, S. and Shekhovtsov, A., "An Improved Vortex Lattice Method for Nonstationary Problems," Journal of Mathematical Sciences, Vol. 104, No. 6, May 2001, pp. 1615-1627.

${ }^{39}$ Wie, S. Y., Lee, S., and Lee, D. J., "Potential Panel and Time-Marching Free-Wake Coupling Analysis for Helicopter Rotor," Journal of Aircraft, Vol. 46, No. 3, May-June 2009, pp. 1030-1041.

${ }^{40}$ Fritz, T. E. and Long, L. N., "Object-Oriented Unsteady Vortex Lattice Method for Flapping Flight," Journal of Aircraft, Vol. 41, No. 6, November-December 2004, pp. 1275-1290.

${ }^{41}$ Leishman, J. G., Principles of Helicopter Aerodynamics, Cambridge Aerospace Series, Cambridge University Press, 2nd ed., 2006.

${ }^{42}$ Lighthill, M. J., "A new approach to thin airfoil theory," The Aeronautical Quarterly, Vol. 3, 1951, pp. 193-210.

${ }^{43}$ Murua, J., Palacios, R., and Graham, J. M. R., "Applications of the Unsteady Vortex-Lattice Method in Aircraft Aeroelasticity and Flight Dynamics," Progress in Aerospace Sciences, 2012, [Commissioned, under review].

${ }^{44}$ Ashley, H., Engineering Analysis of Flight Vehicles, Addison-Wesley Aerospace Series, Addison-Wesley Publishing Co., 1974.

${ }^{45}$ Murua, J., Flexible-Aircraft Dynamics with a Geometricallly-Nonlinear Description of the Unsteady Aerodynamics, Ph.D. thesis, Imperial College London, 2012.

${ }^{46}$ Hodges, D. H., "A Mixed Variational Formulation Based on Exact Intrinsic Equations for Dynamics of Moving Beams," International Journal of Solids and Structures, Vol. 26, No. 11, 1990, pp. 1253-1273.

${ }^{47}$ Patil, M. J., Hodges, D. H., and Cesnik, C. E. S., "Nonlinear Aeroelastic Analysis of Complete Aircraft in Subsonic Flow," Journal of Aircraft, Vol. 37, No. 5, 2000, pp. 753-760.

${ }^{48}$ Simo, J. C. and Vu-Quoc, L., "A Three-Dimensional Finite-Strain Rod Model. Part II: Computational Aspects," Computer Methods in Applied Mechanics and Engineering, Vol. 58, 1986, pp. 79-116. 2001.

${ }^{49}$ Géradin, M. and Cardona, A., Flexible Multibody Dynamics: A Finite Element Approach, John Wiley \& Sons, Inc.,

${ }^{50}$ Cesnik, C. E. S. and Brown, E. L., "Modeling of High Aspect Ratio Active Flexible Wings for Roll Control," $43^{\text {rd }}$ AIAA/ASME/ASCE/AHS/ASC Structures, Structural Dynamics, and Materials Conference, AIAA 2002-1719, Denver, CO, USA, April 2002.

${ }^{51} \mathrm{Su}, \mathrm{W}$. and Cesnik, C. E. S., "Nonlinear Aeroelasticity of a Very Flexible Blended-Wing-Body Aircraft," Journal of Aircraft, Vol. 47, No. 5, 2010, pp. 1539-1553.

${ }^{52}$ Hodges, D. H., "Geometrically Exact, Intrinsic Theory for Dynamics of Curved and Twisted Anisotropic Beams," AIAA Journal, Vol. 41, No. 6, 2003, pp. 1131-1137. 
${ }^{53}$ Sotoudeh, Z. and Hodges, D. H., "Incremental Method for Structural Analysis of Joined-Wing Aircraft," Journal of Aircraft, Vol. 48, No. 5, SeptemberOctober 2011, pp. 1588-1601.

${ }^{54}$ Palacios, R., "Nonlinear Normal Modes in an Intrinsic Theory of Anisotropic Beams," Journal of Sound and Vibration, Vol. 330, No. 8, April 2011, pp. 1772-1792.

${ }^{55}$ Cesnik, C. E. S. and Hodges, D. H., "VABS: A New Concept for Composite Rotor Blade Cross-Sectional Modeling," Journal of the American Helicopter Society, Vol. 42, No. 1, January 1997, pp. 27-38.

${ }^{56}$ Palacios, R. and Cesnik, C. E. S., "Cross-Sectional Analysis of Non-Homogeneous Anisotropic Active Slender Structures," AIAA Journal, Vol. 43, No. 12, 2005, pp. 2624-2638.

${ }^{57}$ Newmark, N. M., "A Method of Computation for Structural Dynamics," ASCE Journal of the Engineering Mechanics Division, Vol. 85, No. 3, 1959, pp. 67-94.

${ }^{58}$ Shearer, C. M. and Cesnik, C. E. S., "Modified Generalized- $\alpha$ Method for Integrating Governing Equations of Very Flexible Aircraft," $4^{7 t h}$ AIAA/ASME/ASCE/AHS/ASC Structures, Structural Dynamics, and Materials Conference, AIAA 2006-1747, Newport, RI, USA, 1-4 May 2006.

${ }^{59}$ Baldelli, D. H., Chen, P. C., and Panza, J., "Unified Aeroelastic and Flight Dynamic Formulation via Rational Function Approximations," Journal of Aircraft, Vol. 43, No. 3, May-June 2006, pp. 763-772.

${ }^{60}$ Zhao, Z. and Ren, G., "Multibody dynamic approach of flight dynamics and nonlinear aeroelasticity of flexible aircraft," AIA A Journal, Vol. 49, No. 1, January 2011, pp. 41-54.

${ }^{61}$ Farhat, C., Lesoinne, M., Stern, P., and Lantéri, S., "High performance solution of three-dimensional nonlinear aeroelastic problems via parallel partitioned algorithms: methodology and preliminary results," Advances in Engineering Software, Vol. 28, 1997, pp. 43-61.

${ }^{62}$ Mukherjee, R. and Gopalarathnam, A., "Post-Stall Prediction of Multiple-Lifting-Surface Configurations Using a Decambering Approach," Journal of Aircraft, Vol. 43, No. 3, 2006, pp. 660-668.

${ }^{63}$ Gogulapati, A. and Friedmann, P. P., "Approximate Aerodynamic and Aeroelastic Modeling of Flapping Wings in Hover

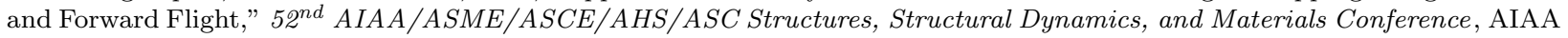
2011-2008, Denver, CO, USA, April 2011.

${ }^{64}$ Murua, J., Palacios, R., and Graham, J. M. R., "A Discrete-Time State-Space Model with Wake Interference for Stability Analysis of Flexible Aircraft," $15^{\text {th }}$ International Forum of Aeroelasticity and Structural Dynamics, IFASD 2011-127, Paris, France, June 2011.

${ }^{65}$ Ypma, T. J., "Historical development of the Newton-Raphson method," SIAM Review, Vol. 37, No. 4, 1995 , pp. 531-551.

${ }^{66}$ Süli, E. and Mayers, D., An Introduction to Numerical Analysis, Cambridge University Press, 2003.

${ }^{67}$ Stengel, R. F., Flight Dynamics, Princeton University Press, 2004.

${ }^{68}$ Cook, M. V., Flight Dynamics Principles: A Linear Systems Approach to Aircraft Stability and Control, Elsevier Ltd, 2nd ed., 2007.

${ }^{69}$ Stevens, B. L. and Lewis, F. L., Aircraft Control and Simulation, John Wiley \& Sons, Inc., 2nd ed., 2003.

${ }^{70}$ Hesse, H., Murua, J., and Palacios, R., "Consistent Structural Linearization in Flexible Aircraft Dynamics with Large Rigid-Body Motion," 53 $3^{\text {rd }}$ AIAA/ASME/ASCE/AHS/ASC Structures, Structural Dynamics, and Materials Conference, Honolulu, HI, USA, April 2012.

${ }^{71}$ Love, M. H., Zink, P. S., Wieselmann, P. A., and Youngren, H., "Body Freedom Flutter of High Aspect Ratio Flying Wings," $46^{\text {th }}$ AIAA/ASME/ASCE/AHS/ASC Structures, Structural Dynamics, and Materials Conference, AIAA 2005-1947, Austin, TX, USA, April 2005.

72 "Broad Agency Announcement (BAA), Vulture II Appendices, Appendix A. 6: Simplified Gust Load Criteria," DARPA TTO, DARPA-BAA-10-04, October 162009.

73 Åström, K. J. and Murray, R. M., Feedback Systems, Princeton University Press, 2008.

${ }^{74}$ Levine, W. S., editor, The Control Handbook, IEEE/CRC Press, 1995, Chapter 52: Automatic Tuning of PID Controllers, by K. J. Åström and T. Hätgglund.

${ }^{75}$ Ziegler, J. B. and Nichols, N. B., "Optimum settings for automatic controllers," ASME Transaction, Vol. 64, 1942, pp. $759-768$.

${ }^{76}$ Cook, R., Palacios, R., Goulart, P., and Roberts, I., "Robust Manoeuvering and Gust Alleviation of Very Flexible Aircraft Using Novel Control Effectors," 15 $5^{\text {th }}$ International Forum of Aeroelasticity and Structural Dynamics, IFASD 2011-047, Paris, France, June 2011.

${ }^{77}$ Zhou, K., Doyle, J. C., and Glover, K., Robust and optimal control, Prentice Hall, 1st ed., 1995. 